\title{
CENTRAL CONFIGURATIONS IN THREE DIMENSIONS
}

\author{
Richard A. Battye ${ }^{1}$, Gary W. Gibbons ${ }^{2}$ and Paul M. Sutcliffe ${ }^{3}$ \\ ${ }^{1}$ Jodrell Bank Observatory, Macclesfield, Cheshire SK11 9DL U.K. \\ \& Department of Physics and Astronomy, Schuster Laboratory, \\ University of Manchester, Brunswick St, Manchester M13 9PL, U.K. \\ Email : rbattye@jb.man.ac.uk \\ 2 Department of Applied Mathematics and Theoretical Physics, \\ Centre for Mathematical Sciences, University of Cambridge, \\ Wilberforce Road, Cambridge CB3 OWA, U.K. \\ Email : G.W.Gibbons@damtp.cam.ac.uk \\ ${ }^{3}$ Institute of Mathematics, \\ University of Kent at Canterbury, \\ Canterbury, CT2 7NZ, U.K. \\ Email : P.M.Sutcliffe@ukc.ac.uk
}

October 2002

\begin{abstract}
We consider the equilibria of point particles under the action of two body central forces in which there are both repulsive and attractive interactions, often known as central configurations, with diverse applications in physics, in particular as homothetic time-dependent solutions to Newton's equations of motion and as stationary states in the One Component Plasma model. Concentrating mainly on the case of an inverse square law balanced by a linear force, we compute numerically equilibria and their statistical properties. When all the masses (or charges) of the particles are equal, for small numbers of points they are regular convex deltahedra, which on increasing the number of points give way to a multi-shell structure. In the limit of a large number of points we argue using an analytic model that they form a homogeneous spherical distribution of points, whose spatial distribution appears, from our preliminary investigation, to be similar to that of a Bernal hard-sphere liquid.
\end{abstract}




\section{Introduction and summary of results}

This is one of a series of papers about central configurations and related problems involving the equilibria of point particles under the action of two-body central forces. The main point of the present work is to survey what is known mathematically from a wide range of disciplines and to link this together with some new, mainly numerical, results of our own, establishing a basis for future work on the subject. Our main emphasis here will be on the classical problem of finding central configurations of particles associated with an inverse square interaction force which are trapped by a linear force, induced by a harmonic potential.

Such models are very common in a wide variety of physical applications, but most of our discussion will focus on systems of gravitating points which in addition to the usual attractive inverse square force, experience a repulsive force proportional to their distance from the origin. They arise naturally when seeking homothetic time-dependent solutions of Newton's equations of motion for gravitating point particles, which in turn may have some relevance to Newtonian Cosmology and models for the large-scale structure of the universe.

Another physical interpretation arises when the inverse square force is thought of as an electrostatic repulsion and the linear force as an attraction, due to a uniform background of the opposite charge. In this guise the problem originally arose in J.J. Thomson's static Plum Pudding model of the atom [26] in which the positive electric charge is smeared out into a uniform ball (the pudding) while the negatively charged electrons correspond to the plums. Although Rutherford's experiments conclusively demonstrated that this model is not relevant as a theory of atomic structure, it nevertheless continues to offer insights into the structure of metals (with the role of positive and negative charges interchanged) and other condensed matter systems and is often referred to as the One Component Plasma (OCP) model [2], or sometimes as classical Jellium.

Central configurations are the critical points of a suitable potential function and those configurations which minimize it are numerically the easiest to study. In fact almost all of this paper will be concerned with central configurations which are local minima that coincide with, or are very close to, the absolute minimum of the potential; only in the case of small numbers of points $(\leq 100)$ will we claim to have found the absolute minima. We use two different numerical techniques to compute these minima. Firstly, a simple multi-start gradient flow algorithm which, given a set of random initial conditions, finds the path of steepest descent toward a local minimum. The other technique is that of simulated annealing [31], which uses thermal noise to deter the system from falling into a local minimum which is not the global one. We used these two methods in tandem to increase our confidence in finding the true minimum for small numbers of points and to find a stationary point close to the true minimum for larger numbers. By running the codes many times when the number of points is large, we were able to deduce that there are very many local minima with energies close to the absolute minimum. In this regard it resembles related problems such as that of placing point charges on a sphere and those of sphere packing. 
It turns out that the Plum Pudding interpretation provides the key to understanding the properties of central configurations for moderate and large numbers of points and is also quite valuable for understanding the solutions for small numbers of points. The idea is that for many purposes one may envisage the equilibria as a packing of Thomson-type hydrogen atoms, that is, electrically neutral spheres containing a single negative charge at the centre in a shell of positive charge.

More quantitatively the spheres correspond to the Thomson atoms described above. The fact that this correspondence may be elevated to a precise quantitative tool was apparently first recognized by Leib and Narnhoffer [20] who used it to obtain a rigorous lower bound for the energy of the OCP in terms of a close packing of Thomson atoms. Our numerical results show that the actual minimum is incredibly close to the Leib-Narnhoffer bound and leads to a picture of the equilibria not unlike Bernal's random close packing model of liquids [3]. We use the word liquid deliberately because despite the wide-spread belief that in the limit of infinite numbers of particles the minimum of the OCP model is given by a Body Centred Cubic (BCC) crystal, our preliminary results for up to 10,000 particles appear to show no sign of crystallization, nor long range translational order. They are, however, crudely consistent with a Bernal liquid.

A second piece of intuition which appears to be useful is to consider points uniformly distributed inside a sphere. Remarkably, by using the continuum limit, an analytic expression can be derived for the probability distribution for separations in terms of the radius of the confining sphere, which is known in terms of the number of points. This two-point function provides an analytic test of the homogeneity of the distribution, which is passed with considerable accuracy. It is also possible to compute a three-point statistic associated with the distribution of triangles, and we find agreement there too.

We will present our results for various values of the number of points, $N$, in three groups designed to exemplify the specific characteristics of the solutions:

(I) Small numbers of points, $N \leq 100$ say.

(II) Moderate numbers of points, say $100<N<1000$.

(III) Large numbers of points. Here we are able to deal with $1000 \leq N \leq 10,000$.

For the most part we will stick to the case where all the masses (charges) of the particles are equal $\left(m_{1}=m_{2}=. .=m_{N}=m\right)$.

A summary of the results is as follows :

In case (I) we claim to have found the absolute minima by using the two different algorithms with a wide range of different initial conditions. For $N \leq 12$ the points lie at the vertices of a polyhedron which is a deltahedron (one made entirely from triangles) except for the antiprism found for $N=8$, and is regular if $N=4,6$ or 12 . The polyhedron is a tetrahedron if $N=4$, an octahedron if $N=6$ and an icosohedron if $N=12$. When $N=13$ the minimum is a single point surrounded by the other twelve in an icosahedral structure and for $13 \leq N \leq 57$ and $N=60$ there are effectively two shells. There is a link between 
$N=13$ being the first value at which a point is found inside the polyhedron and the fact that at most 12 spheres of equal radius can touch a given sphere of the same radius. For $58 \leq N \leq 100$ (except $N=60$ which is a particularly symmetric structure) there are three shells.

In case (II) the configurations found by our algorithms, which are local minima but may not be the absolute minima, look at first glance to be roughly uniform. However, closer examination of the precise distribution of points reveals a clearly defined system of shells. For example, if one plots the density as a function of radius it oscillates around uniformity with a regular period. Each of the shells appears to have roughly the same surface density and the radii of the shells appear to be in arithmetic progression. This leads to an approximate description of the number of points in each shell. As the number of points increases the minimum of the energy comes closer and closer to the lower bound, suggesting that the assumptions under which it is derived provide a good picture of the distribution of the particles.

In case (III) we see that a clear spatial uniformity of the distribution emerges. This is exemplified by computing two-point and three-point statistics and comparing them to the continuum description of the problem. With a few minor caveats related to the discreteness of the distribution, we find remarkable agreement between the analytic expressions and those found for large $N$; the results for the values $N=1000$ and $N=10,000$ will be presented. This uniformity of the density distribution is a consequence of Newton's theorem: for an inverse square law, the force due to a spherically symmetric distribution of matter is the same as if the total mass is concentrated at the centre of mass. This is not the case for any other force law. Of considerable interest is the spatial distribution of the particles in these uniform distributions. We computed the distribution of the distance between nearest neighbours and found it to be sharply peaked, suggesting that each particle can be thought of as a sphere of fixed radius and that they may pack as in the classical sphere packing problem. However, a preliminary investigation of the angular distribution of nearest neighbours reveals no evidence of long-range orientational order as one might expect, for example, in a solid. The main caveat to this result is that for large values of $N$ we are unable to have much confidence in having found the global minimum of the energy. Nonetheless, the asymptotic approach to the lower bound on the energy suggests that the configurations we have found are very close to the global minimum.

\section{Central configurations and related problems}

\subsection{Definition of the problem}

Classically, central configurations are defined as sets of $N$ points $\mathbf{r}_{a} \in \mathbb{R}^{3}, a=1, \ldots, N$ satisfying

$$
\frac{\Lambda}{3} m_{a} \mathbf{r}_{a}+\sum_{b \neq a} \frac{G m_{a} m_{b}\left(\mathbf{r}_{b}-\mathbf{r}_{a}\right)}{\left|\mathbf{r}_{a}-\mathbf{r}_{b}\right|^{3}}=0
$$


where the constants $G$ and $\Lambda$ are both strictly positive, for a set of strictly positive masses $\left(m_{1}, \ldots m_{N}\right)$. The constant $G$ may be thought of as Newton's constant, in which case, the constant $\Lambda$ has the dimensions of (time) ${ }^{-2}$. It follows that the centre of mass of the configuration lies at the origin

$$
\sum_{a} m_{a} \mathbf{r}_{a}=0 .
$$

It is convenient to divide (2.1) by $m_{a}$ and write it as

$$
\frac{\Lambda}{3} \mathbf{r}_{a}+\sum_{b \neq a} \frac{G m_{b}\left(\mathbf{r}_{b}-\mathbf{r}_{a}\right)}{\left|\mathbf{r}_{a}-\mathbf{r}_{b}\right|^{3}}=0 .
$$

Equation (2.1) may be interpreted as stating that each mass point is in equilibrium under the action of a repulsive radial force proportional to the mass and the distance from the origin and the gravitational attraction of the remaining points. The repulsive force is such as arises in theories with a cosmological constant $\Lambda$. It also arises naturally if one makes a time-dependent homothetic ansatz in Newton's equations of motion. One may instead think of repulsive Coulomb forces between the particles and an attraction to the origin. This attraction can arise from a uniform density of charge with opposite sign to that of the particles. This will be discussed in detail later in Section 2.3.

To begin with we shall show how to eliminate the apparent origin dependence and replace the first term by a sum of two-body repulsions proportional to the separation $r_{a b}=\left|\mathbf{r}_{a}-\mathbf{r}_{b}\right|$. If we define the total mass $M$ by

$$
M=\sum_{a} m_{a}
$$

then

$$
m_{a} \mathbf{r}_{a}=\frac{1}{M} \sum_{b \neq a} m_{a} m_{b}\left(\mathbf{r}_{a}-\mathbf{r}_{\mathbf{b}}\right)+\frac{m_{a}}{M} \sum_{b} m_{b} \mathbf{r}_{b} .
$$

Using (2.2) and (2.5) in (2.1) we obtain

$$
\sum_{b \neq a} \mathbf{F}_{a b}=0,
$$

where

$$
\mathbf{F}_{a b}=m_{a} m_{b}\left(\mathbf{r}_{a}-\mathbf{r}_{b}\right)\left(\frac{\Lambda}{3 M}-\frac{G}{r_{a b}^{3}}\right) .
$$

Note that (2.7) is invariant under translation of the points and, while all solutions of (2.1) are solutions of (2.7), these latter solutions can have any centre of mass. We shall only be interested in solutions centred on the origin since any solution not centred on the origin can be obtained from one that is by translation.

Clearly a particular inter-particle distance is picked out, that is,

$$
r_{a b}=R=\left(\frac{3 G M}{\Lambda}\right)^{\frac{1}{3}} .
$$


Two particles a distance $R$ apart feel no mutual force.

Note that in units in which $G=\frac{\Lambda}{3}=1$, which we shall use from now on, and if $m_{1}=m_{2}=\ldots=m_{N}=1$, the distance at which the force vanishes is $R=N^{\frac{1}{3}}$. The first few values are

$$
1.259921, \quad 1.4422496, \quad 1.5874011, \quad 1.7099759 .
$$

Thus every side of the solutions associated with the dipole, triangle and tetrahedron are given by the first three values respectively. In the case $N=5$ we get a triangular bi-pyramid (see Section 3.3). This cannot be regular, but the last value is an estimate for the average separation. If one believes that the forces essentially saturate after roughly this distance one gets a close packing model with diameter roughly 1.7. In fact, as we shall see later, this is a slight overestimate and the numerical data suggests the diameter $d \approx 1.65$.

To gain a further insight into the significance of the radius $R$, consider a very large number of points in a roughly spherically symmetric configuration centred on the origin and in which the total mass enclosed within a sphere of radius $r$ is $M(r)$. By Newton's celebrated theorem, the attractive force per unit mass exerted on a thin shell of radius $r$ depends only on the masses enclosed within the shell and is given by

$$
\frac{G M(r)}{r^{2}} \text {. }
$$

This is an estimate for the second term in (2.3). The cosmic repulsion, i.e. the first term in $(2.3)$ is

$$
\frac{\Lambda}{3} r,
$$

and, therefore, equating these two expressions gives

$$
M(r)=\frac{\Lambda}{3 G} r^{3} .
$$

It follows that any roughly spherically symmetric configuration will occupy a ball of radius $R$ with roughly uniform density. We shall see later that for large numbers of points this uniformity holds with high accuracy.

Note that the argument given above applies only for an inverse square force law. Thus, we do not expect spatial uniformity for other force laws and indeed we do not find it to be the case (see Section 6).

\subsection{Potential functions}

Solutions of (2.1) are critical points of the function

$$
V=V_{-1}+V_{2},
$$

where

$$
V_{-1}=\sum_{a} \sum_{b<a} \frac{G m_{a} m_{b}}{\left|\mathbf{r}_{a}-\mathbf{r}_{b}\right|}
$$


which is homogeneous degree -1 and

$$
V_{2}=\frac{\Lambda}{6} \sum_{a} m_{a} \mathbf{r}_{a}^{2}
$$

which is homogeneous degree 2. Euler's theorem then gives the virial relation

$$
V_{-1}=2 V_{2}
$$

Of course, because the system is rotationally invariant, the critical points are not isolated, they have 3 rotational zero modes.

One could instead look at critical points of (minus) the gravitational potential energy $V_{-1}$ and regard $\frac{\Lambda}{3}$ as playing the role of a Lagrange multiplier fixing the value of the function

$$
I=\frac{1}{2} \sum_{a} m_{a} \mathbf{r}_{a}^{2}
$$

In what follows we shall refer to solutions as stable if they are absolute minima of $V$, as metastable if they are local minima and unstable if the Hessian has some negative eigenvalues. The terminology is most appropriate for the electrostatic problem since for the gravitational problem the appropriate potential function is minus $V$. However, the issue of dynamical stability is more complicated in that case as we shall discuss in detail in our future paper on the cosmological interpretation of our results.

Finally we remark that, at the expense of introducing three translational zero modes, one may replace the quadratic potential $V_{2}$ by

$$
\tilde{V}_{2}=\frac{\Lambda}{6 M} \sum_{a<b} m_{a} m_{b}\left(\mathbf{r}_{a}-\mathbf{r}_{b}\right)^{2}
$$

Thus we need to extremize a sum of two body potentials

$$
\sum_{a<b} m_{a} m_{b} U\left(r_{a b}\right)
$$

where

$$
U(r)=\frac{G}{r}+\frac{\Lambda}{6 M} r^{2}
$$

\subsection{One Component Plasma and Thomson's plum pudding}

The One Component Plasma (OCP) [2], sometimes called the classical Jellium model, is essentially the same problem as originally studied by Thomson [27] as a model of the atom. Nowadays it is often used as a model for metals at high density in which one assumes that quantum mechanically degenerate electrons provide a uniform background of negative charge in which there are immersed positively charged nuclei. Of course in Thomson's original model the roles of positive and negative charges are reversed. 
Note that the problem of placing point charges on a sphere (see Section 2.8) is often, but mistakenly, referred to as the Thomson problem. For the Thomson problem (or equivalently the OCP, since the sign of the charges is irrelevant here) one considers a uniformly positively charged domain $\Omega \subset \mathbb{R}^{3}$ with volume $A$ containing $N$ negatively charged corpuscles. The sum of the negative charges is taken to be equal to the total positive charge. The potential energy of the system is taken to consist of three parts

$$
V_{O C P}=V_{--}+V_{+-}+V_{++}
$$

$V_{--}$is the positive mutual electrostatic energy of the negatively charged particles. $V_{+-}$ is the electrostatic potential energy of the negative charges in the potential generated by the uniformly distributed positive background. Finally, one includes the potential energy, $V_{++}$, of the uniformly charged positive background.

Usually one takes all of the charges to have the same value, but one may consider the case when they differ. If one does so one obtains a system identical to the one discussed in Sections 2.1 and 2.2. Rather than introducing further unnecessary notation we shall continue with our present conventions leaving to the reader the trivial task of transcription to the electrostatic units of his or her choice (ref. [24] may prove useful in this respect).

With the proviso that all particles must lie inside $\Omega$, we have that

$$
\begin{gathered}
V_{--}=V_{-1} \\
V_{+-}=-G M \sum_{a}\left(\frac{m_{a}}{A} \int \frac{d^{3} \mathbf{r}}{\left|\mathbf{r}-\mathbf{r}_{\mathbf{a}}\right|}\right),
\end{gathered}
$$

and

$$
V_{++}=\frac{G M^{2}}{2}\left(\frac{1}{A^{2}} \iint \frac{d^{3} \mathbf{r} d^{3} \mathbf{r}^{\prime}}{\left|\mathbf{r}-\mathbf{r}^{\prime}\right|}\right)
$$

In the case when $\Omega$ is taken to be a ball of radius $R$ we can evaluate the integrals.

$$
\begin{gathered}
V_{+-}=-\frac{3 G M^{2}}{2 R}+\frac{G M}{2 R^{3}} \sum m_{a} \mathbf{r}_{a}^{2} \\
V_{++}=\frac{3}{5} \frac{G M^{2}}{R} .
\end{gathered}
$$

Thus

$$
V_{O C P}=V_{-1}+\frac{3 G M}{\Lambda R^{3}} V_{2}-\frac{9 G M^{2}}{10 R} .
$$

Evidently in the case that $\Omega$ is a ball of radius $R$, the critical points that are the equilibria of $V_{O C P}$ and $V$ coincide as long as we set

$$
\frac{3 G M}{\Lambda}=R^{3}
$$

but the values of $V_{O C P}$ and $V$ at the critical points will differ. In the case that $\Omega$ is not a ball, even the critical points will differ. 


\subsection{Upper and lower bounds for the minimum of the energy}

The following rigorous bounds, whose proofs are discussed in the following two subsections, constrain the minimum value of the energy

$$
\frac{9}{10} N\left(N^{\frac{2}{3}}-1\right) \leq V^{\min } \leq \frac{9}{10} N(N-1)^{\frac{2}{3}}
$$

They are a valuable check on our numerical results, and it turns out that the lower bound is a particularly good estimate for the actual minimum energy. For large numbers of particles our numerical results support the conjecture that there are many local minima with energies very close to the lower bound.

\subsubsection{An upper bound}

The minimum value of a function can never be greater than the average value of the function over any sub-domain of its domain. Let us apply this principle to $V$ which is a function on $\mathbb{R}^{3 N}$ and consider its average value with respect to the uniform distribution over $\left(B_{3}\left(R_{0}\right)\right)^{N}$ the product of $N$ balls of radius $R_{0}$, that is, we average over the sub-domain $0 \leq\left|\mathbf{r}_{a}\right| \leq R_{0}$. For a pair of particles, and if $n>-3$ the Williamson average (see Section $2.6)$ is

$$
\left\langle r_{i j}^{n}\right\rangle=\frac{72\left(2 R_{0}\right)^{n}}{(n+3)(n+4)(n+6)},
$$

and thus

$$
\left\langle V_{-1}\right\rangle=\frac{6 G}{5 R_{0}} \sum_{b<a} m_{a} m_{b} .
$$

On the other hand

$$
\left\langle V_{2}\right\rangle=\frac{\Lambda R_{0}^{2}}{10} \sum m_{a}
$$

Therefore, the upper bound for the minimum value of $V^{\text {min }}$ is, assuming that $\Lambda=3, G=$ $1, m_{a}=1$,

$$
\frac{3}{10} N R_{0}^{2}+\frac{3}{5 R_{0}} N(N-1)
$$

The upper bound will be optimal, that is, smallest, if we choose $R_{0}^{3}=(N-1)$. Substituting back we get

$$
V^{\min } \leq \frac{9}{10} N(N-1)^{\frac{2}{3}}
$$

\subsubsection{A lower bound for the energy}

As explained in [2], Leib and Narnhoffer [20] proved a rigorous lower bound for $V_{O C P}$, at least in the case that $m_{1}=m_{2}=\ldots=m_{N}=m$. One defines an ion radius $a$ by

$$
N \frac{4 \pi}{3} a^{3}=A
$$


Thus in the case that $\Omega$ is a sphere of radius $R$

$$
a=\frac{R}{N^{\frac{1}{3}}} .
$$

Note that in our solutions $a \approx 1$ with considerable accuracy. Now one has the extensive lower bound:

$$
V_{O C P} \geq-N \frac{9}{10} \frac{G m}{a} .
$$

The interpretation is that the right hand side of (2.37) is the energy of $N$ non-overlapping spheres of radius $a$ with total charge zero, in other words of $N$ non-overlapping Thomson type Hydrogen atoms. The packing of these atoms plays an important role in determining the distribution of the points.

We may re-write the Leib-Narnhoffer bound (setting $G=m=a=1$ ) as

$$
V^{\min } \geq \frac{9}{10} N^{\frac{5}{3}}-N \frac{9}{10}
$$

\subsection{Continuum limit}

This has already been alluded to above. It is most easily obtained by replacing the discrete distribution of masses by a continuous density distribution

$$
\sum m_{a} \delta\left(\mathbf{x}-\mathbf{r}_{a}\right) \longrightarrow \rho(\mathbf{x})
$$

in the variational problem. Ignoring self-energies, we therefore need to extremize

$$
\frac{1}{2} G \iint \rho(\mathbf{x}) \rho(\mathbf{y}) \frac{1}{|\mathbf{x}-\mathbf{y}|} d^{3} x d^{3} y+\frac{\Lambda}{6} \int \mathbf{x}^{2} \rho(\mathbf{x}) d^{3} x+\lambda \int \rho(\mathbf{x}) d^{3} x,
$$

where $\lambda$ is a Lagrange multiplier enforcing the constraint that the total mass

$$
M=\int \rho(\mathbf{x}) d^{3} x
$$

is fixed. Variation of the density gives a linear integral equation for $\rho$

$$
G \int d^{3} y \rho(\mathbf{y}) \frac{1}{|\mathbf{x}-\mathbf{y}|}+\frac{\Lambda}{6} \mathbf{x}^{2}+\lambda=0 .
$$

Acting on this equation with the Laplacian gives

$$
-4 \pi G \rho+\Lambda=0 .
$$

We have recovered our previous result that the density must be constant. But it is clear that the density cannot be everywhere constant and still satisfy the constraint that the total mass be fixed. Moreover we have not deduced that the boundary of the blob of uniform fluid must be spherically symmetric. This is presumably because we have not been sufficiently careful about boundary effects in the variation. 


\subsection{Separation probability distribution}

The cumulative probability for the separation of two points $\mathbf{r}_{1}$ and $\mathbf{r}_{2}$ uniformly distributed inside a sphere of radius $R$ seems to have been given originally by Williamson using an extremely ingenious geometrical argument [32]. Below we rederive this result using differential forms.

The volume form on $\mathbb{R}^{3} \times \mathbb{R}^{3}$ is given in spherical polars about some fixed axis with origin $O$ by

$$
\omega=r_{1}^{2} \sin \theta_{1} d r_{1} \wedge d \theta_{1} \wedge d \phi_{1} \wedge r_{2}^{2} \sin \theta_{2} d r_{2} \wedge d \phi_{2}
$$

Consider the triangle $O 12$ with sides of length $r_{1}, r_{2}$ and $r_{12}$. Let $\psi$ be the angle $O 12$ and $\chi$ the angle of the plane of the triangle about an axis along the side $O 1$. Then by means of a rotation of the second set of spherical polars one has

$$
\omega=r_{1}^{2} \sin \theta_{1} d r_{1} \wedge d \theta_{1} \wedge d \phi_{1} \wedge r_{12}^{2} \sin \psi d r_{12} \wedge d \psi \wedge d \chi .
$$

Now the cosine formula for the triangle tells us that

$$
r_{2}^{2}=r_{1}^{2}+r_{12}^{2}-2 r_{1} r_{12} \cos \psi
$$

and therefore

$$
r_{2} d r_{2}=\left(r_{1}-r_{12} \cos \psi\right) d r_{1}+\left(r_{12}-r_{1} \cos \psi\right) d r_{12}+r_{1} r_{12} \sin \psi d \psi .
$$

Eliminating $d \psi$ gives

$$
\omega=r_{1} r_{2} r_{12} \sin \theta_{1} d r_{1} \wedge d r_{2} \wedge d r_{12} \wedge d \theta_{1} \wedge d \phi_{1} \wedge d \chi
$$

The integrals over $\theta_{1}, \phi_{1}, \chi$ may be done immediately so that

$$
\omega=8 \pi^{2} r_{1} r_{2} r_{12} d r_{1} \wedge d r_{2} \wedge d r_{12}
$$

In order to obtain $d P$ we set $r=r_{12}$ and integrate over $r_{1}$ and $r_{2}$ consistent with the points 1 and 2 being confined to lie inside a ball of radius $R$ and divide by $16 \pi^{2} R^{6} / 9$. To perform the integration it is convenient to introduce the coordinates $x=r_{1}+r_{2}$ and $y=r_{1}-r_{2}$. The ranges of integration are obtained by applying the triangle inequalities and are given by $r \leq x \leq 2 R-|y|$ and $|y| \geq r$.

The result is

$$
\operatorname{Prob}\left(\left|\mathbf{r}_{1}-\mathbf{r}_{2}\right| \leq r\right)=\frac{r^{3}}{R^{3}}-\frac{9 r^{4}}{16 R^{4}}+\frac{r^{6}}{32 R^{6}}
$$

One has of course $\operatorname{Prob}\left(\left|\mathbf{r}_{1}-\mathbf{r}_{2}\right| \leq 2 R\right)=1$. The probability density is thus

$$
d P=p(r) d r=\left(\frac{3 r^{2}}{R^{3}}-\frac{9 r^{3}}{4 R^{4}}+\frac{3 r^{5}}{16 R^{6}}\right) d r
$$

from which, the mean separation is

$$
\langle r\rangle=\int_{0}^{2 R} r p(r) d r=\frac{36}{35} R \approx 1.02857 R .
$$


The numerical results described later agree with this rather well. In what follows we shall denote averages with respect to the Williamson distribution as above and averages taken over our numerically generated set of points (or pairs of points in this case) by an overbar. Thus numerically, as we shall show, we find that $\bar{r} \approx\langle r\rangle$ to a good accuracy. Of course to compare we must say what the value of $R$ is. This will usually be done using the

formula $R=(N-1)^{\frac{1}{3}}$. Recall that this relation between $R$ and $N$ is the one derived in Section 2.4.1 in order to make the upper bound on $V^{\text {min }}$ optimal.

The most likely separation is given by the root between $r=0$ and $r=2 R$ of the cubic equation

$$
6-\frac{27 r}{4 R}+\frac{15 r^{3}}{16 R^{3}}=0 .
$$

Because $r=2 R$, is a root, the cubic factorizes

$$
(r-2 R)\left(5 r^{2}+10 R r-16 R^{2}\right)=0,
$$

and the solution we want is

$$
r=\frac{R}{5}(\sqrt{105}-5) \approx 1.04939 R .
$$

\subsection{Distribution of triangles}

Later we shall present the statistics of triples of points computed numerically. For points uniformly distributed inside a sphere an interesting quantity to consider is the distribution of angles over all triangles given by any three points. Unfortunately, it appears that no analytic expression for this distribution of angles is known. Deriving a formula for this distribution would therefore seem to be a very worthwhile exercise in geometric probability. One result that is known is that the probability that any angle is acute is given by $33 / 70[32$, 15]. Numerically we shall find a good agreement with this value.

\subsection{Point charges on a sphere}

The problem here is to minimize the potential energy $V_{-1}$ subject to the constraint that the points lie on a sphere of some given radius. For reasons which are unclear to us, this problem has come to be associated with Thomson's name even though he appears not to have posed it explicitly. What he had in mind is perhaps that given the existence of a shell structure then one only needs to minimize the energy with respect to positions inside the shell. A large number of papers have investigated this problem; see [7] and references therein for details.

To see this more explicitly, note that in order to enforce the constraint one introduces $N$ Lagrange multipliers $\Lambda_{a}$. One obtains the equations

$$
\frac{\Lambda_{a}}{3} m_{a} \mathbf{r}_{a}+\sum_{b \neq a} \frac{G m_{a} m_{b}\left(\mathbf{r}_{b}-\mathbf{r}_{a}\right)}{\left|\mathbf{r}_{a}-\mathbf{r}_{b}\right|^{3}}=0 .
$$


The interpretation of the first term in (2.56), $\mathbf{F}_{a}=\frac{\Lambda_{a}}{3} m_{a} \mathbf{r}_{a}$ is that it is the inward force exerted on the particle necessary to counteract the outward repulsion of the remaining particles. Thus on solving the equations and constraints, the Lagrange multipliers $\Lambda_{a}$ will turn out to be positive. If it happens that all the $\Lambda_{a}$ 's are equal then this is also a central configuration (it is a solution of (2.1)). This may be true only approximately if the distribution of points is sufficiently spherically symmetric.

\subsection{Sphere packing problems}

As we have indicated above, there appears to be a close relation between central configurations and the classical sphere packing problem: to prove that there is no packing of congruent spheres in three dimensions with density or packing ratio $\eta$ exceeding that of a face-centred cubic (FCC) with $\eta=\pi / \sqrt{18} \approx 0.74048$. This long-standing conjecture, due originally to Harriot and Kepler has now been proved by Hales (see ref. [14] for an overview and references).

The highest packing density is achieved for FCC packing which is crystallographic, but it is well-known that there are uncountably many other packings, both crystallographic and non-crystallographic with the same packing density. Thus, viewed as an optimization problem, the sphere packing problem has infinitely many optima with essentially the same density. Moreover local optima with vacancies, that is with a finite number of isolated spheres missing, have in the infinite limit the same density. In the case of finite sphere packings there will clearly be many local optima very close to the closest packing. This feature is certainly shared by central configurations. The comparison of central configurations with sphere packings can be taken further. For example, a key fact about any sphere packing is, as stated first in print by Halley [16] in connection with his prior account of Olber's Paradox, that at most 12 congruent spheres may touch a thirteenth congruent sphere. In other words, the maximum coordination number (that is the number of nearest neighbours) for close-packing is 12 . This fact, asserted by Newton and denied by Gregory [12], would be a useful diagnostic tool in assessing whether our configurations are closepacked (they are certainly not FCC) but unfortunately for central configurations there is no unambiguous way to define a coordination number, and any numerical results computed are very sensitive to its definition.

One may refine the above discussion a little [21]. The local cell for FCC packing is a rhombic dodecahedron. However, the local cell of smallest volume is a regular pentagonal dodecahedron. This cannot, because of it's five-fold symmetry, give a lattice packing of course but it can appear in small clusters and this happens in our case for 13 particles. In the same note it is remarked that most physicist's believe that the optimum for the One Component Plasma is a body-centred cubic (BCC) packing. As we discuss in Section 5.2 we have seen little evidence for that in our results. It is perhaps worth remarking here that the published energies of various lattices in the One Component Plasma problem [9] seem to be extremely close and this alone indicates it shares with the sphere packing problem the feature that there are many critical points very close to the minimum. It turns out to be worth exploring in more detail some further features of sphere packings since they 
have some diagnostic value in understanding our numerical results. This is especially true in connection with the shell structure which will be discussed in Section 4.

\section{Case I : small numbers of points}

Small numbers may be studied analytically and numerically; historical information may be found for example in ref. [33] or ref. [13], and we largely ignore planar solutions since (for $N>3$ ) these appear to be unstable. By symmetry, one expects any regular polyhedron to provide a solution but not necessarily a stable one. One can also place a mass point at the centre of a regular polyhedron. For the same reason it is also clear that pyramidal and bi-pyramidal solutions should exist for arbitrary numbers of particles as well as prism and anti-prism solutions. Again, placing a mass point at the centre of bi-pyramids, prisms and anti-prisms is possible. According to Hagihara [13], Blimovitch [4, 5] claims two similar and similarly situated regular polyhedra are possible, as well as a regular polyhedron together with its dual.

\subsection{N=3 Lagrange's triangle}

Relative equilibria are planar solutions of (2.1) and include collinear solutions. They may also give rise to rigidly rotating solutions of Newton's equations of motion. Planar configurations will be the subject of another paper and so here we will restrict attention to the case when $N=3$. In that case, for arbitrary masses, the only non-collinear solution is Lagrange's equilateral triangle. In standard units the sides of the triangle are ${ }^{3} \sqrt{ } 3=1.4422496 \ldots$ which is larger than the distance ${ }^{3} \sqrt{ } 2=1.259921 \ldots$ of the dipole. In what follows it will be useful to envisage Lagrange's solution as three spheres touching one another. For some interesting recent work on the planar case including the relation to a hard disc model and with applications to the final shapes of systems of particles moving under repulsive inverse square law forces, see refs. [10, 11]. For other work on planar configurations see ref. [17]. If one really were dealing with two dimensions, then the analogous problem would involve a logarithmic potential; for results on this case see ref. [18].

\section{$3.2 \mathrm{~N}=4$ : tetrahedral configuration}

The first non-planar case is for $N=4$. The existence of a regular tetrahedral solution for arbitrary positive values of $\left(m_{1}, m_{2}, m_{3}, m_{4}\right)$ was shown by Lehmann Filhés in 1891 [19] and the uniqueness among all non-planar solutions by Pizetti in 1903 [22].

The existence is obvious by noting that if we choose side length $(3 G M \Lambda)^{\frac{1}{3}}$ for our tetrahedron then by (2.8) every two body force will vanish. The necessity follows by noting that if the four are not co-planar, then the six inter-particle distances $r_{a b}, 1 \leq a<b \leq 4$ give six independent coordinates on $C_{4}\left(\mathbb{R}^{3}\right) / E(3)$ and so the potential function must be stationary with respect to independent variations of all six inter-particle distances. From 


\begin{tabular}{|c|c|c|}
\hline Multiplicity & Valency & Edge Lengths \\
\hline 3 & 4 & $1.545(2), 1.872(2)$ \\
2 & 3 & $1.545(3)$ \\
\hline
\end{tabular}

Table 1: For the $N=5$ polyhedron we list the multiplicity of each type of vertex, its valency, and its edge lengths together with their multiplicities (given as the number in brackets after each edge length).

(2.18) and (2.20), it follows that every inter-particle distance must be a stationary point of the function $U$ in $(2.20)$.

In normalized units the side of the tetrahedral configuration, which should be envisaged as four mutually touching close packed spheres is ${ }^{3} \sqrt{ } 4=1.5874011 \ldots$ The significance of the tetrahedron as far as our work is concerned is that it not infrequently seems to occur as a sub-configuration inside a nested set of shells.

\section{$3.3 \quad \mathrm{~N}=5$ : triangular bi-pyramid}

Surprisingly this is not completely understood [25, 8]. Numerically one finds a minimum in the form of triangular bi-pyramid. In addition one knows that there is a solution with one point at the centre of a tetrahedron and a pyramidal solution on a square base [25, 8]. It is not difficult to imagine other, presumably unstable, solutions.

The bi-pyramid is not regular. However, it closely resembles a bi-pyramidal cluster obtained by close-packing 5 equal spheres. The three points which form the equilateral triangle are at a distance of 1.081 from the origin, whereas the two remaining points are at a distance of 1.104 from the origin. In terms of edges lengths we can summarize this information in Table 1. For each type of vertex we give its multiplicity (the number of times such a vertex occurs in the configuration), its valency (the number of nearest neighbours), and the edge lengths of the polyhedron given by the distances of the nearest neighbours. The numbers in round brackets after each edge length denote the multiplicity of this nearest neighbour length. Note that each edge of the polyhedron is represented twice, since we deal with each vertex individually.

The information in Table 1 therefore summarizes the fact that there are three 4-valent vertices (the ones which form the equilateral triangle) and two 3 -valent vertices (the ones which sit above and below the equilateral triangle). The equilateral triangle has edge length 1.872 but the six remaining edge lengths are all shorter at 1.545. Taking the average of the nine edges lengths gives $\bar{l}=1.654$, which is in good agreement with the diameter $d=1.65$ which we use in our sphere packing model.

It is interesting to note that the same triangular bi-pyramid also arises as the energy minimizing configuration using a scale invariant energy function [1] and the ratio of the two distances from the origin $1.081 / 1.104=0.979$ is precisely the same value as obtained in that case. In fact for all $N \leq 12$ the configurations of minimizing points appear to be remarkably similar for the two problems (taking into account the scale invariance of one of the energy functions). 


\begin{tabular}{|c|c|c|}
\hline Multiplicity & Valency & Edge Lengths \\
\hline 5 & 4 & $1.509(2), 1.790(2)$ \\
2 & 5 & $1.790(5)$ \\
\hline
\end{tabular}

Table 2: Vertex types and edge lengths for the $N=7$ polyhedron.

\begin{tabular}{|c|c|c|}
\hline Multiplicity & Valency & Edge Lengths \\
\hline 3 & 4 & $1.615(4)$ \\
6 & 5 & $1.615(2), 1.742(2), 1.989(1)$ \\
\hline
\end{tabular}

Table 3: Vertex types and edge lengths for the $N=9$ polyhedron.

\section{$3.46 \leq N \leq 12$}

In this range the minima form a single shell. If $N=6$ we have an octahedron, with edge length 1.676. If $N=7$ we have a pentangular bi-pyramid. The five points forming the pentagon sit on a circle of radius 1.283 and the remaining two points are at a distance of 1.248 from the origin. The ratio of these two distances $1.283 / 1.248=1.028$ is again equal to that for the pentangular bi-pyramid which results from minimizing the scale invariant energy function of ref. [1]. In terms of edge lengths this information is summarized in Table 2. The average edge length is $\bar{l}=1.696$.

$N=8$ is the first example in which some of the faces are not triangular, it being a square anti-prism, obtained from a cube by rotating the top face by $45^{\circ}$ relative to the bottom face. Each vertex is 4-valent and contains two edges of length 1.581 and two of length 1.738, giving an average length $\bar{l}=1.660$.

For $N=9$ the points lie on the vertices of three parallel equilateral triangles, with the middle triangle rotated by $60^{\circ}$ relative to the other two. The edge lengths are given in Table 3 and the average is $\bar{l}=1.705$.

The $N=10$ polyhedron can be obtained from the $N=8$ one by replacing each square by a hat made from four triangles with a 4 -valent vertex. The edge lengths are given in Table 4 and the average is $\bar{l}=1.706$.

For $N=11$ the polyhedron contains a vertex with six nearest neighbours. The existence of the single vertex with six neighbours means that this configuration is not very symmetric. The edge lengths are given in Table 5 and the average is $\bar{l}=1.680$.

$N=12$ forms a regular icosahedron with edge length $\bar{l}=1.682$.

We have already commented that these configurations occur as the minima of a scale invariant energy function and furthermore, as discussed in that situation [1], the associated

\begin{tabular}{|c|c|c|}
\hline Multiplicity & Valency & Edge Lengths \\
\hline 8 & 5 & $1.600(1), 1.621(2), 1.898(2)$ \\
2 & 4 & $1.600(4)$ \\
\hline
\end{tabular}

Table 4: Vertex types and edge lengths for the $N=10$ polyhedron. 


\begin{tabular}{|c|c|c|}
\hline Multiplicity & Valency & Edge Lengths \\
\hline 1 & 6 & $1.524(2), 1.977(4)$ \\
2 & 5 & $1.624(2), 1.730(2), 1.825(1)$ \\
2 & 5 & $1.624(2), 1.659(1), 1.806(2)$ \\
4 & 4 & $1.511(1), 1.546(1), 1.730(1), 1.806(1)$ \\
2 & 4 & $1.524(1), 1.546(2), 1.659(1)$ \\
\hline
\end{tabular}

Table 5: Vertex types and edge lengths for the $N=11$ polyhedron.

polyhedra are of the same combinatoric type as those associated with the solution of the points on a sphere problem discussed in Section 2.8. In fact, the correspondence is more than a combinatoric match since a projection of the points onto the sphere appears to produce the solutions of the sphere problem.

In fig. 1 we display our configurations of points, for $3 \leq N \leq 12$, by plotting spheres of diameter $d=1.65$ around each of the $N$ points. This highlights the similarity to sphere packing configurations.

\subsection{Deltahedra}

A regular deltahedron is a polyhedron all of whose faces are equilateral triangles. A combinatoric deltahedron is a polyhedron of the same combinatoric type. For any deltahedron we have $2 E=3 F$ (in the following $E, F, V$ refer to the number of edges, faces and vertices of a polyhedron). If it has the topology of a sphere we have $F-E+V=2$, and thus

$$
F=2(V-2), \quad E=3 V-2 \text {. }
$$

There are just 8 convex regular deltahedra. They have $V=4,5,6,7,8,9,10,12$. There is no convex deltahedron with $V=11$.

The minimum energy solutions for $N=4,5,6,7,9,10,12$ closely resemble (or are) regular deltahedra, as can be seen from the tables of edge lengths. In each of these cases there are no more than three different edge lengths forming the polyhedron and they are all reasonably close in value. This is related to the geometry of deltahedra taken together with the existence of Lagrange's triangular solution and the fact that a particular spacing is picked out at which the inter-particle force vanishes.

The forces on regular convex deltahedra are almost in equilibrium and presumably only require small adjustments to cancel exactly. The fact that the $N=11$ configuration is not regular is automatic, since, as mentioned above, no regular deltahedron exists with 11 vertices. From Table 5 it can be seen that there are many different edge lengths forming the $N=11$ polyhedron.

The actual polyhedra themselves are displayed in fig. 2 for $4 \leq N \leq 12$. 


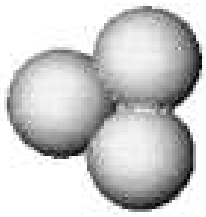

3

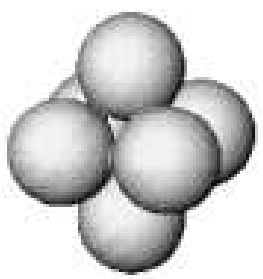

6

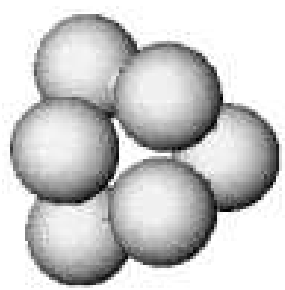

9

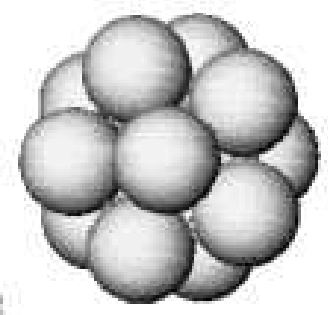

12

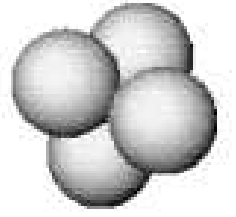

4

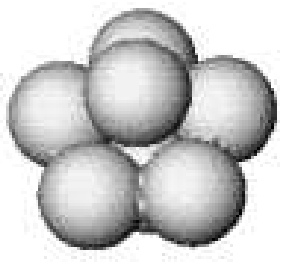

7

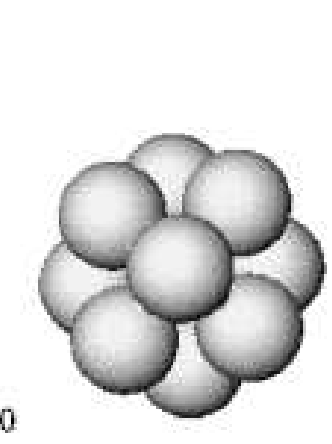

\section{0}

5
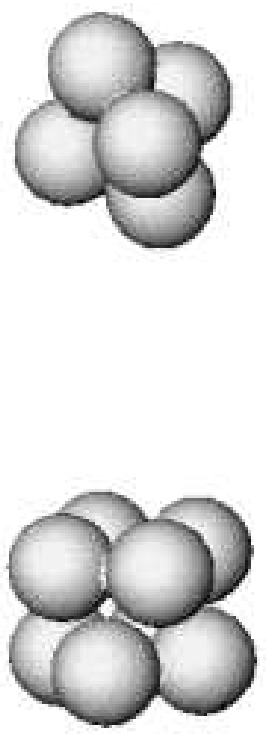

8

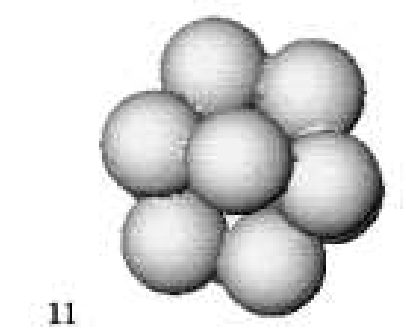

Figure 1: For $3 \leq N \leq 12$ we display our configurations of $N$ points by plotting spheres of diameter $d=1.65$ around each of the points.

See fig1.jpg for a colour version of this figure. 


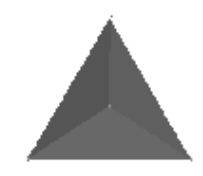

4

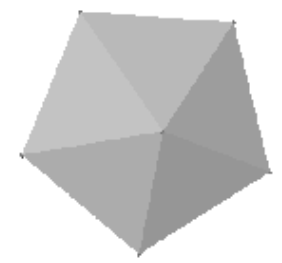

7

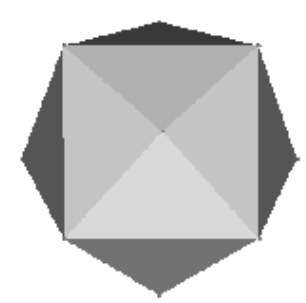

10

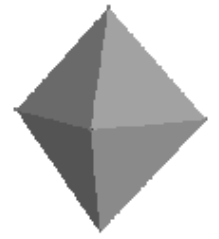

5

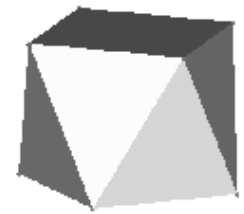

8

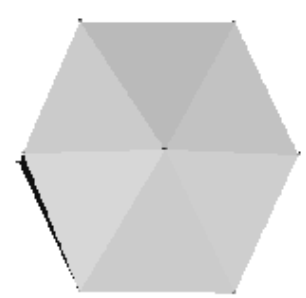

11

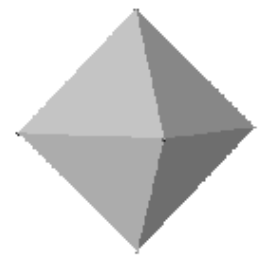

6

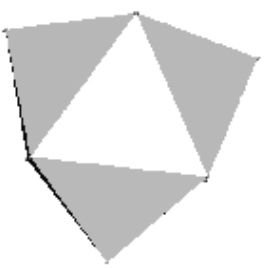

9

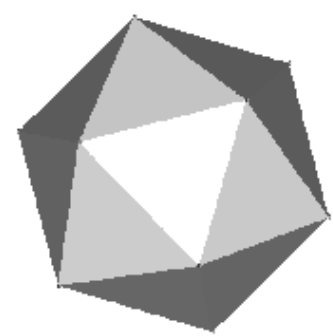

12

Figure 2: The polyhedra associated with the set of $N$ points for $4 \leq N \leq 12$. 


\begin{tabular}{|c|c|c|c|c|c|c|c|}
\hline$N$ & $V$ & $V /$ Bound & Shells & $N$ & $V$ & $V /$ Bound & Shells \\
\hline 1 & 0.0000 & 1.000000 & $/ / 00 / 00$ & 51 & 586.13 & 1.001364 & $10 / 41 / 00$ \\
\hline 2 & 1.1906 & .126006 & $2 / 00 / 00$ & 52 & 606.0110 & 1.001339 & $10 / 42 / 00$ \\
\hline 3 & 01 & 19 & & 53 & 69 & & $10 / 43 / 00$ \\
\hline 4 & 5.6696 & 036227 & $=/ 00 / 00$ & 54 & 646.5716 & 1.001305 & $10 / 44 / 00$ \\
\hline 5 & 8.9100 & 1.029100 & $5 / 00 / 00$ & 55 & 667.2312 & 1.001261 & $12 / 43 / 00$ \\
\hline 6 & 12.6391 & 016787 & $/ 00 / 00$ & 56 & 688.1384 & 1.001197 & $12 / 44 / 00$ \\
\hline 7 & 17.0243 & 1.016157 & 7/00/00 & 57 & 709.3484 & 1.001 & $12 / 45 / 00$ \\
\hline 8 & 21.8643 & 1.012 & $/ 00 / 00$ & 58 & 730.8185 & 1.00 & $01 / 12 / 45$ \\
\hline 9 & 27.2144 & 1.009937 & /00/00 & 59 & 752.5386 & 1.001178 & $01 / 12 / 46$ \\
\hline 10 & 33.0575 & 1.008642 & $/ 00 / 00$ & 60 & 774.5108 & 1.001159 & $12 / 48 / 00$ \\
\hline 11 & & & & 61 & 796.7202 & & $01 / 12 / 48$ \\
\hline 12 & 46.0883 & 1.006 & $/ 00 / 00$ & 62 & 819.2150 & 1.00 & $01 / 13 / 48$ \\
\hline 13 & 53.3116 & 1.00 & $/ 12 / 00$ & 63 & 841.9375 & 1.0 & $01 / 14 / 48$ \\
\hline 14 & 60.9584 & 59 & & 64 & 864.9324 & & $1 / 14 / 49$ \\
\hline 15 & 68.9 & 1.00 & 0 & 65 & 888. & 1.0 & $01 / 14 / 50$ \\
\hline 16 & 16 & & & 66 & & & $01 / 15 / 50$ \\
\hline 17 & 86.2009 & 20 & $/ 10$ & 67 & 935.3691 & & $01 / 15 / 51$ \\
\hline 18 & 4178 & 9 & 00 & 68 & 90 & 1.0 & $01 / 16 / 51$ \\
\hline 19 & 5 & & & 69 & & & $01 / 16 / 52$ \\
\hline 20 & .0418 & 1.00 & 0 & 70 & 008.0 & 1.0 & $01 / 16 / 53$ \\
\hline 21 & & & & 71 & & & 54 \\
\hline 22 & 9 & & 0 & 72 & 37 & & $01 / 17 / 54$ \\
\hline 2 & 15 & 0 & $2 / 21$ & 73 & & & $01 / 17 / 55$ \\
\hline 24 & 7 & & 0 & 74 & & & $01 / 17 / 56$ \\
\hline 25 & .4147 & 3 & $/ 2$ & 75 & 75 & & $01 / 18 / 56$ \\
\hline 26 & & & & 76 & & & $8 / 57$ \\
\hline 27 & 1 & & 0 & 77 & & & $/ 58$ \\
\hline 28 & & 11 & & 78 & 97 & & $01 / 18 / 59$ \\
\hline 29 & 2 & & & 79 & & & $1 / 18 / 60$ \\
\hline 30 & 7 & & & 80 & & & $/ 59$ \\
\hline 1 & & & & 81 & 292.9 & & $01 / 20 / 60$ \\
\hline 32 & 81 & $\$ 5$ & 0 & 82 & 33 & & $02 / 20 / 60$ \\
\hline 33 & 276.4994 & & & 83 & 94 & & $02 / 21 / 60$ \\
\hline 34 & 97 & & & 84 & & & $02 / 21 / 61$ \\
\hline 35 & 2 & & & 85 & & & $02 / 21 / 62$ \\
\hline 36 & 36 & & & 86 & & & $02 / 21 / 63$ \\
\hline 37 & 4 & 9 & 0 & 87 & 94 & & $02 / 22 / 63$ \\
\hline 38 & 33 & & & 88 & & & $02 / 22 / 64$ \\
\hline 39 & .2331 & & & 89 & 78 & 1.0 & $03 / 22 / 64$ \\
\hline 40 & & & & 90 & & & $03 / 22 / 65$ \\
\hline 41 & .5671 & 88 & & 91 & 576.3 & & $03 / 23 / 65$ \\
\hline 42 & 6643 & 1.00 & 00 & 92 & 1605.9 & 1.0 & $03 / 22 / 67$ \\
\hline 43 & & & & 93 & & & $03 / 24 / 66$ \\
\hline 44 & & & & 94 & & & $03 / 24 / 67$ \\
\hline 45 & 472.6332 & & & 95 & 1695. & 1.0 & $04 / 24 / 67$ \\
\hline 46 & & & & 96 & 94 & & $04 / 24 / 68$ \\
\hline 47 & 9 & & & 97 & 1757 & & $04 / 25 / 68$ \\
\hline 48 & & & & 98 & 1787.9348 & & $04 / 25 / 69$ \\
\hline 49 & .1978 & 1.00 & & 99 & 1819.( & & $04 / 26 / 69$ \\
\hline 50 & 6.5327 & 1.001394 & 09/41/00 & 100 & 1850.3349 & 1.000727 & $04 / 26 / 70$ \\
\hline
\end{tabular}

Table 6: For $N \leq 100$ we list the minimum energy $V$, the ratio of this energy to the lower bound, and the number of points in each shell. 


\section{6 $N \leq 100$}

In Table 6 we present the minimal value of the energy for all $N \leq 100$. The ratio of this energy to the value of the lower bound (2.29) is also given, from which it is clear that the lower bound is an extremely tight one and that the percentage excess over this bound decreases with increasing $N$. These results were obtained using both a multi-start simulated annealing algorithm and a multi-start gradient flow code. Both methods were applied independently and led to the same common results. We therefore believe that the configurations we have found are the global minima for each value of $N$ and the energies are accurate to the level quoted. As expected, during our computations we found large numbers of local minima, which we shall ignore. The same codes were used to generate the configurations discussed later in the paper with $N \gg 100$, but we make no claim that they are the global minima, merely that they are local minima (which may or may not be global) whose energies we expect to be very close to that of the global minimum.

For $N \leq 12$ all the points lie close to the surface of a sphere, but this is not the case for $N>12$. In particular for $N=13$ there are 12 points on the vertices of a regular icosahedron and an additional single point at the origin. We denote this structure by the code $01 / 12$, indicating that there are two shells, the first one containing a single point and the second containing 12 points. For $N \leq 100$ there are at most three shells. In Table 6 we present the shell structure for the minimal energy configuration by listing its code as above. We find that within each shell the arrangement of points resembles that for the solution of the problem of Section 2.8. For example, if an inner shell contains four points then they are located on the vertices of a regular tetrahedron.

In fig. 3 we plot the ratio of the energy to the bound for $30 \leq N \leq 100$. From this plot we see that there are magic numbers at which this ratio drops more sharply than usual. The most striking examples are $N=32$ and $N=38$. These magic numbers occur when two (or more) shells both have a large symmetry group. For $N=32$ we see from Table 6 that the shell structure is $4 / 28$, that is, there are two shells with the inner shell containing 4 points and the outer shell containing 28 points. The solution of the sphere problem for 4 points is a regular tetrahedron, while the solution of the sphere problem for 28 points also has tetrahedral symmetry. These two solutions, if appropriately aligned as inner and outer shells can therefore preserve tetrahedral symmetry, and this is precisely the arrangement we find for the $N=28$ configuration. In fig. 4 we plot the distance of each of the 32 points from the origin. We see that within the second shell there is a substructure consisting of three mini-shells, each of which contains a multiple of four points, consistent with the tetrahedral symmetry. A similar situation arises for $N=38$, with the shell structure being 6/32. The solution of the sphere problem for 32 points has icosahedral symmetry, the associated polyhedron being the dual of the truncated icosahedron, while for 6 points

the solution of the sphere problem is an octahedron. The $N=38$ solution consists of these two nested solutions aligned to preserve their common tetrahedral subgroups. 


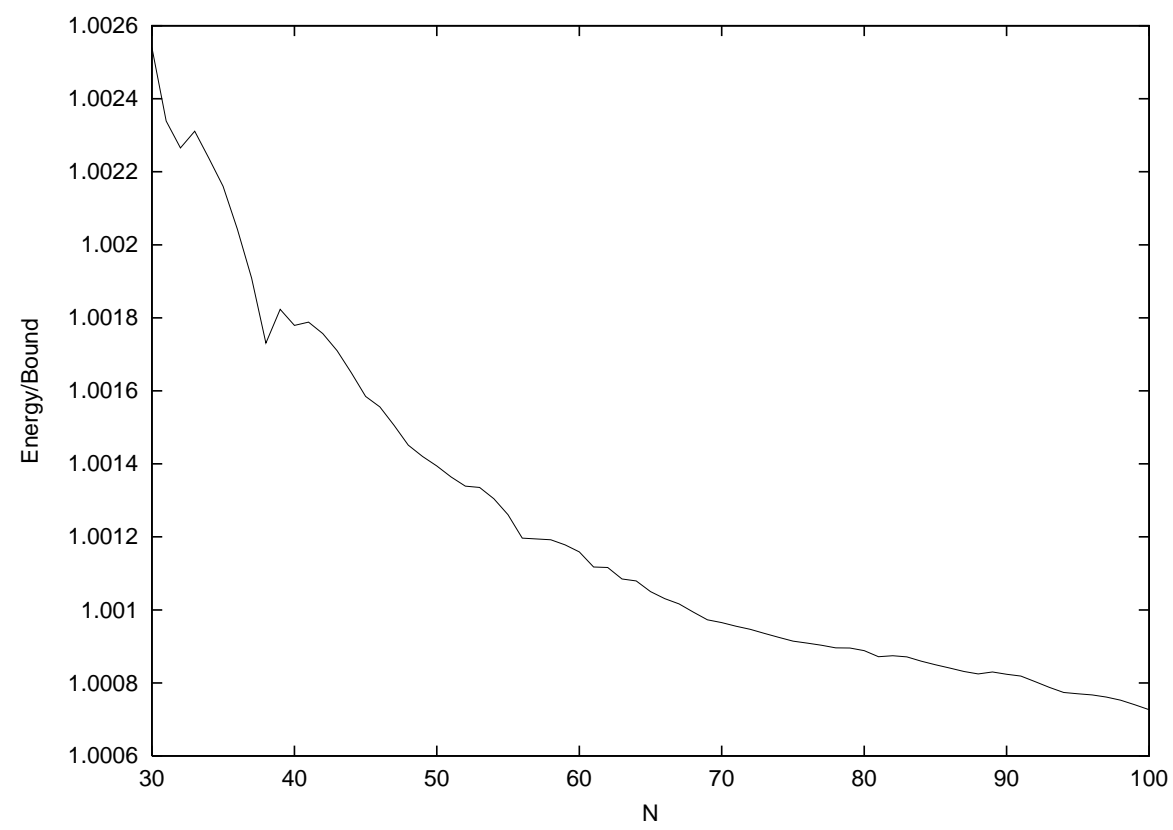

Figure 3: The ratio of the energy to the bound for $30 \leq N \leq 100$.

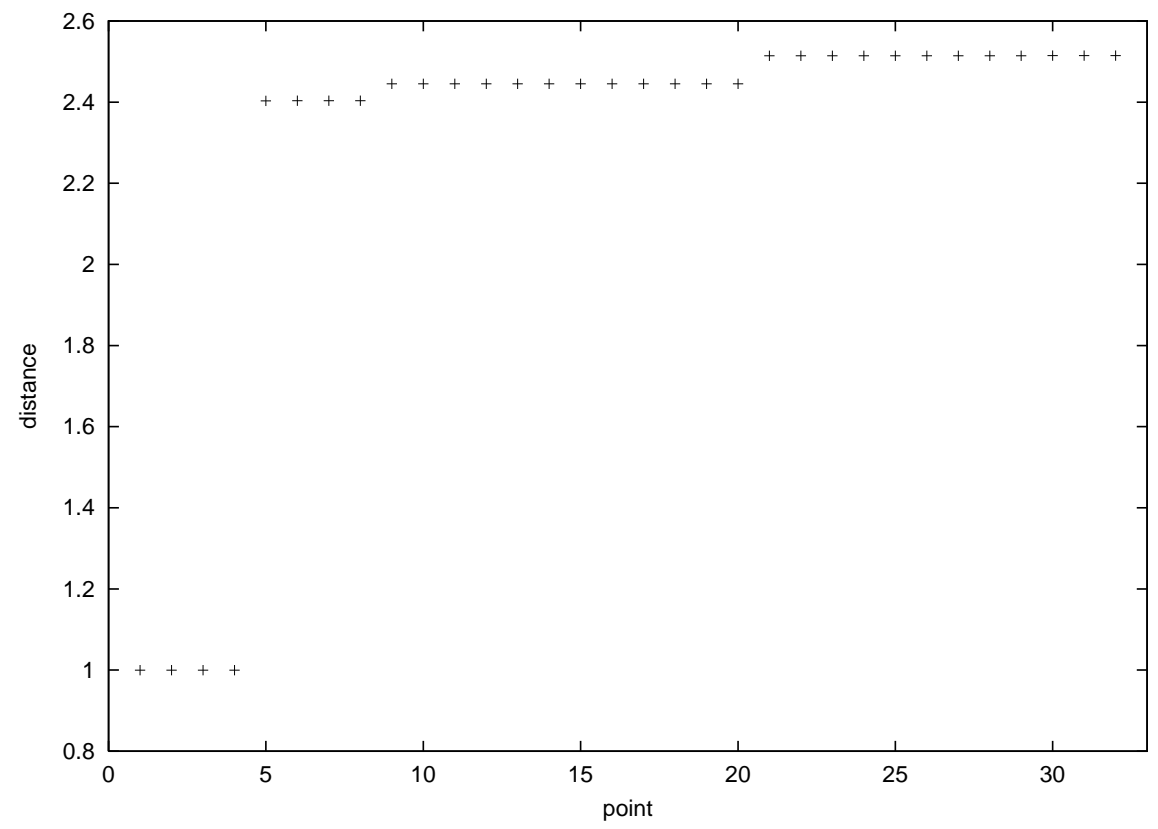

Figure 4: The distance of each point from the origin for the $N=32$ minimal energy configuration. 


\section{Case II : moderate numbers of points}

We have discussed in the previous Section that there is a transition from a single shell when $N=12$ to two when $N=13$, and that a similar transition, from two to three shells, takes place on passing from $N=57$ to $N=58$. One might expect that further transitions take place as one increases $N$ and indeed this is the case, although as we will discuss in the subsequent Section this eventually gives way to what is effectively a uniform distribution for $N>1000$. Here, we will discuss and quantify the structure of the shells.

\subsection{Shell structure}

Considerations based on Newton's theorem make it plausible that, at least for moderately large numbers of points, one may expect solutions made up of nested shells, each one giving an approximate solution to the problem of Section 2.8 and arranged in such a way that the average density of points is uniform. More precisely if $M_{k}$ is the total mass within the $k$ 'th shell which has radius $R_{k}$ then

$$
3 G M_{k}=\Lambda R_{k}^{3}
$$

In what follows it will be convenient to count the shells from the centre so that if there are $S$ shells, the last shell has radius $R_{S}$ and $M_{S}=M$. The mass of the $k^{\prime}$ th shell is

$$
\Delta M_{k}=M_{k}-M_{k-1}
$$

and thus

$$
\Delta M_{k}=\frac{\Lambda}{3 G}\left(R_{k}^{3}-R_{k-1}^{3}\right)
$$

We can analogously define

$$
\Delta N_{k}=N_{k}-N_{k-1},
$$

where $N_{k}$ is the number of particles inside and on the $k$ 'th shell. For each shell we can define the surface density to be $\sigma_{k}=\Delta N_{k} /\left(4 \pi R_{k}^{2}\right)$.

Our numerical calculations suggest that, ignoring a single particle or pairs of particles which might congregate at the centre, the radii of the shells are in arithmetic progression and that the surface density of particles in each shell is approximately constant; the relevant constants being almost universal between different configurations. Let us define the constants $R_{\mathrm{c}}$ and $R_{0}$ such that $R_{k} \approx k R_{\mathrm{c}}-R_{0}$ and set $\sigma_{k} \approx \sigma$. Then $\Delta N_{k} \approx 4 \pi \sigma R_{k}^{2}$ and hence for large $k$ we see that $\Delta N_{k} \propto k^{2}$. This provides an interesting approximation which appears to have some veracity if one ignores the innermost shells. Moreover, one can use the virialization condition (2.16) to give

$$
V \approx \frac{3}{2} \sum_{i=1}^{S} R_{i}^{2} \Delta N_{i} \approx 6 \pi \sigma \sum_{i=1}^{S} R_{i}^{4} \approx 6 \pi \sigma \sum_{i=1}^{S}\left(i R_{\mathrm{c}}-R_{0}\right)^{4} .
$$

This estimate for the energy, while nowhere near as accurate as that discussed in Section 2.4 , is accurate to within a few percent. 


\begin{tabular}{|c|c|c|c|c|}
\hline$k$ & $\Delta N_{k}$ & $R_{k}$ & $\delta R_{k}$ & $4 \pi \sigma_{k}$ \\
\hline 1 & 6 & 1.227 & 0.029 & 3.983 \\
2 & 31 & 2.655 & 0.150 & 4.399 \\
3 & 78 & 4.148 & 0.150 & 4.533 \\
4 & 149 & 5.678 & 0.129 & 4.622 \\
5 & 236 & 7.197 & 0.037 & 4.556 \\
\hline
\end{tabular}

Table 7: The shell structure for a configuration with $N=500$ points. Note that the radii of the shells are approximately in arithmetic progression and that the value of $\sigma_{k}$ is roughly constant.

\subsection{Worked example}

To illustrate the discussion of the previous Section, here we will consider a specific example with $N=500$ points. The configuration may not necessarily be that of minimal energy, but $V=27903.2=1.00018 B_{500}$ where $B_{N}=\frac{9}{10} N\left(N^{\frac{2}{3}}-1\right)$ denotes the lower bound given by (2.29). The structure of the solution is illustrated in fig. 5 and fig. 6. Fig. 5 is created in a similar way to fig. 1 by surrounding each point by a sphere of diameter $d=1.65$. On the right is a slice through the centre of the figure on the left with the spheres in different shells coloured differently. It is clear that there are 5 shells. This is further illustrated by fig. 6 where the distance from the origin of each point is plotted. Notice that the shells are distinct in that there are obvious gaps between them.

Table 7 lists the values of $\Delta N_{k}, R_{k}$ and $\sigma_{k}$ for each of the shells. Included also is $\delta R_{k}$, the standard deviation of the radii of the particles in each shell, which shows that the inner shells are much less localized than the outer crust. This can also be seen from fig. 6 . Except for the innermost shell $\sigma \approx 4$.5. The data values for $R_{k}$ are plotted in fig. 7 together with the linear fit $R_{k}=k R_{\mathrm{c}}-R_{0}$ using the values $R_{\mathrm{c}}=1.5$ and $R_{0}=0.31$. Using these values, and the fact that there are 5 shells, the final formula in (4.5) gives the estimate $V \approx 27315$, illustrating that this approximation is accurate to within a few percent.

\subsection{Rough estimates}

In this Section we describe how to obtain some rough estimates of the inter-particle distance, the surface density, and the number of particles in each shell.

A regular tetrahedron has a height $\sqrt{3} / 2$ times the length of a side. Thus an estimate for the inter-particle distance is

$$
d \approx \frac{2}{\sqrt{3}}\left(R_{k}-R_{k-1}\right) \approx 2 R_{\mathrm{c}} / \sqrt{3} .
$$

Using the earlier value of $R_{\mathrm{c}}=1.5$ yields

$$
d \approx 1.73
$$

which is not out of line with the absence of inter-particle forces at very small and very large separations. 


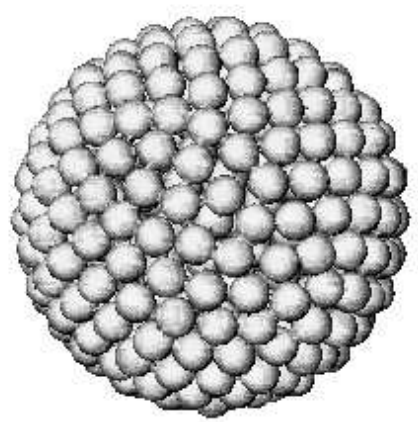

a

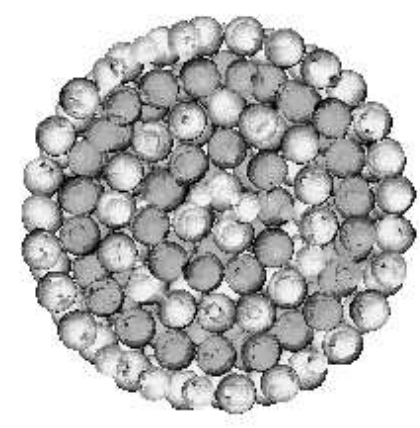

b

Figure 5: A configuration with 500 points. On the left are all the points realized using the same method as in fig. 1 . On the right is a slice through the centre with alternate shells having different colours.

See fig5.jpg for a colour version of this figure.

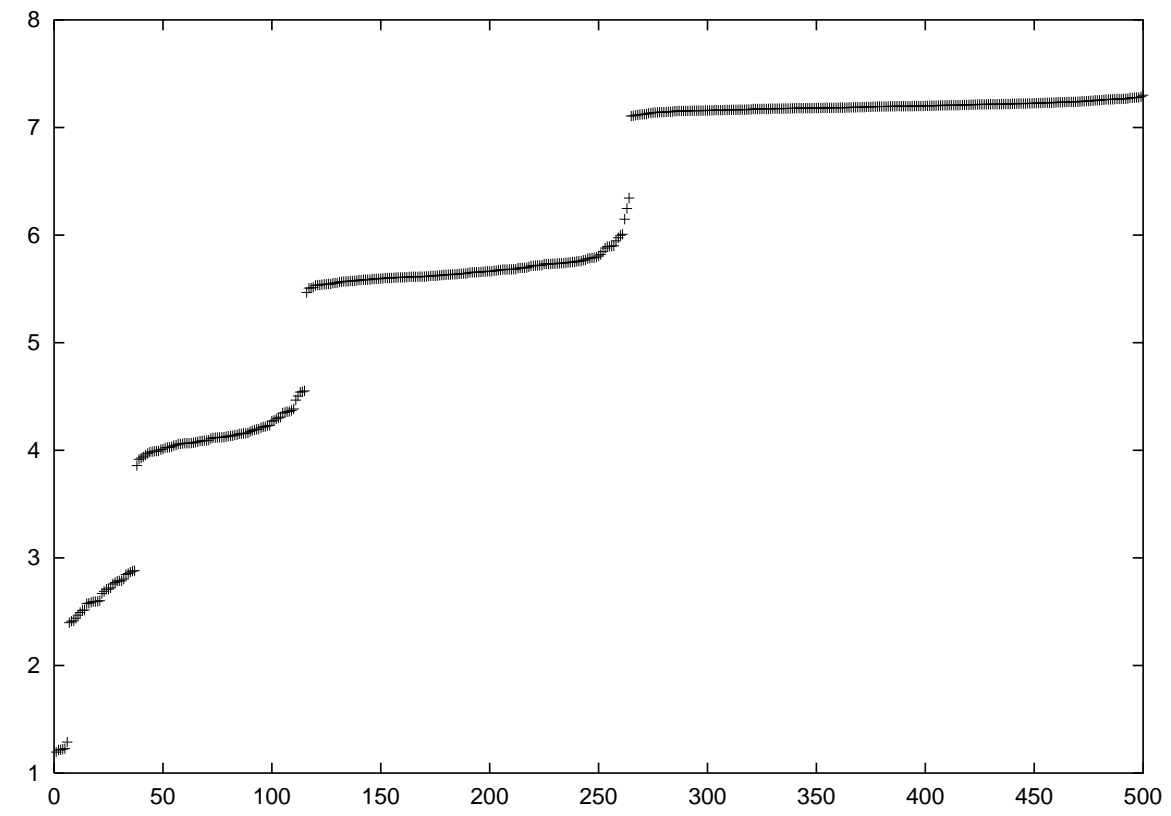

Figure 6: The distance of each point from the origin for $N=500$ 


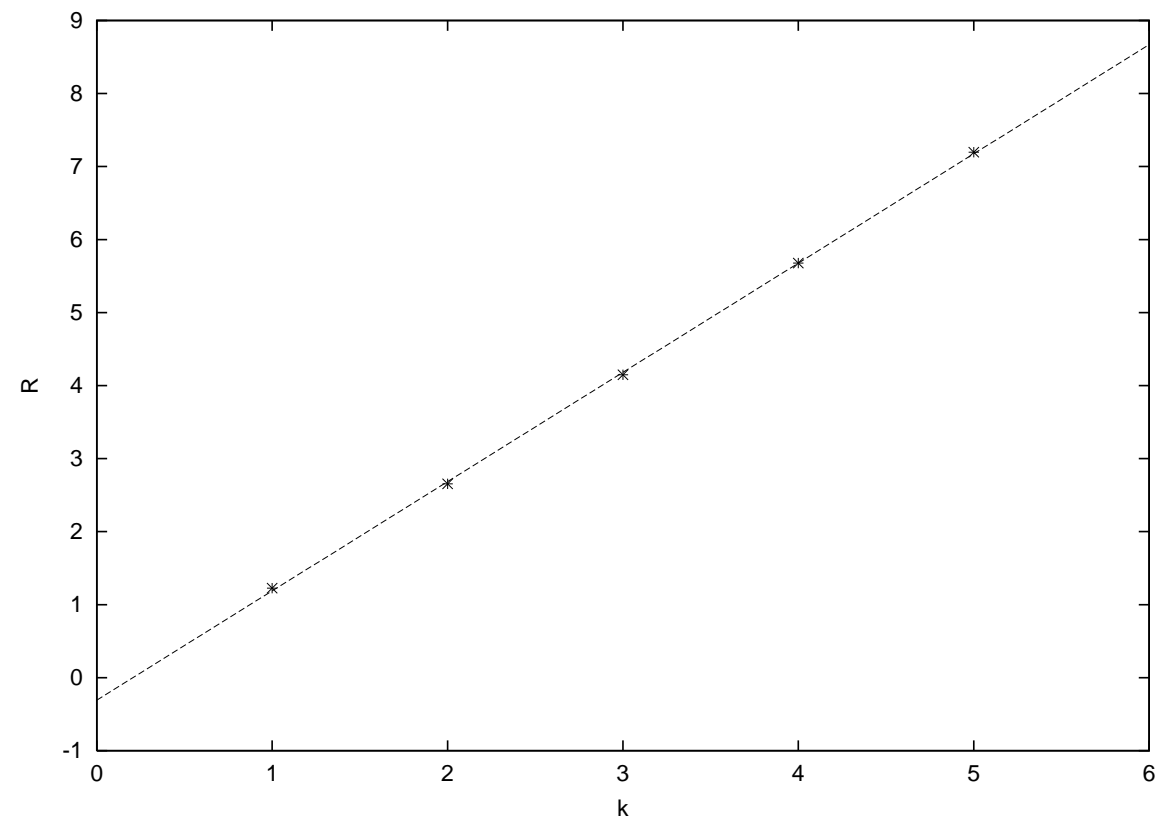

Figure 7: The shell radii $R_{k}$ together with a linear fit, for an $N=500$ configuration containing 5 shells.

To get a handle on the surface density we note that the closest packing for circles on the plane (a problem originally tackled by Kepler and by Harriot) is attained for hexagonal packing for which the surface packing ratio is

$$
\zeta=\frac{\pi}{2 \sqrt{3}}=0.9068996 \ldots
$$

A rough estimate for the number of spheres of diameter $d$ that can be packed in a sphere of radius $R_{k}$ is thus

$$
\Delta N_{k} \approx \frac{4 \pi R_{k}^{2} \zeta}{\pi\left(\frac{d}{2}\right)^{2}} .
$$

Thus we get the estimate (better an upper bound)

$$
4 \pi \sigma \approx \frac{\Delta N_{k}}{R_{k}^{2}} \approx \frac{16 \zeta}{d^{2}}
$$

Substituting $d \approx 1.73$ yields

$$
4 \pi \sigma_{k} \approx 4.85
$$

which is certainly larger than 4.5 but not enormously so.

We can obtain a crude over-estimate for the number of particles in each shell by replacing $R_{k}$ in (4.9) by the approximation $R_{k} \approx k R_{\mathrm{c}}$, where we have neglected the negative 


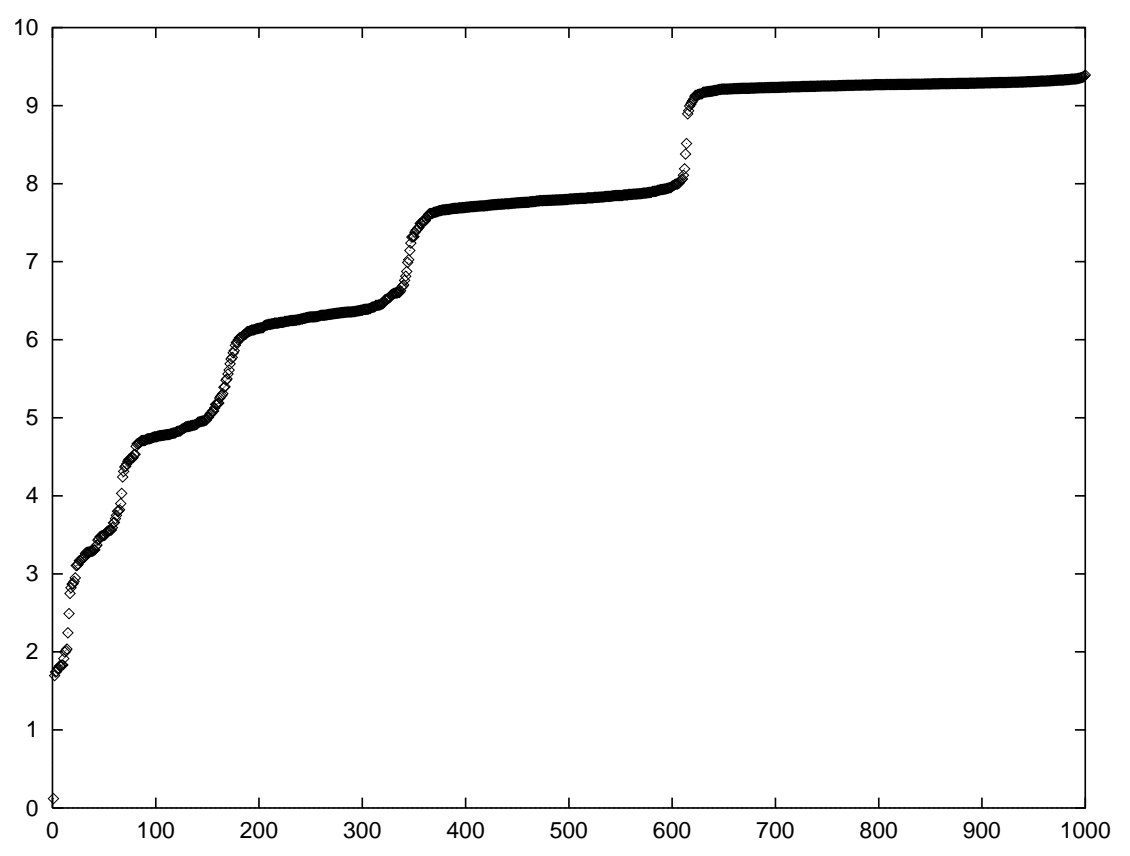

Figure 8: The distance of each point from the origin for $N=1000$.

constant term in our earlier linear fit (the source of the over-estimation). Then using the final relation in (4.6) we arrive at

$$
\Delta N_{k} \approx 2 \pi \sqrt{3} k^{2} \approx 10.88 k^{2} .
$$

Taking the integer part of this expression produces the values $\Delta N_{1}=10, \Delta N_{2}=43, \Delta N_{3}=$ $97, \Delta N_{4}=174, \Delta N_{5}=272$ which should be compared with those in Table 7 . We see that these numbers are indeed over-estimates but give reasonable ball-park values.

\section{Case III : large numbers of points}

\subsection{Statistical results: close packing and hard sphere model}

The following statistical results are based on the lowest energy configuration of 1000 points that we were able to compute. This configuration has an energy $V=1.000103 B_{1000}$, where $B_{N}=\frac{9}{10} N\left(N^{\frac{2}{3}}-1\right)$ denotes the lower bound given by (2.29). Thus, although we are not able to claim that this is the global minimum energy configuration, its energy is clearly very close to that of the global minimum because of its small deviation from the lower bound. In fig. 8 we plot the distance of each point from the origin for $N=1000$. This plot demonstrates that for 1000 points there is still a shell-like structure, associated with the visible steps, but the distinction between the shells is now quite blurred. Fig. 9 displays the 


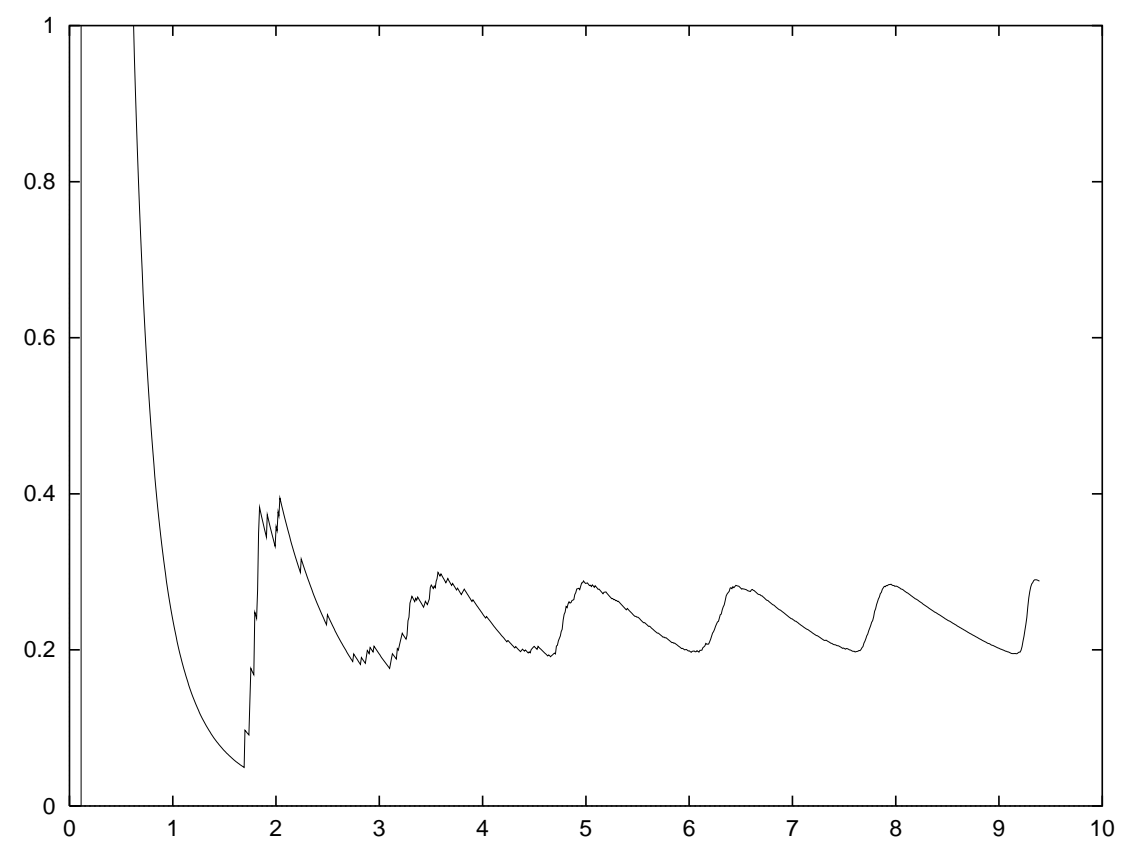

Figure 9: The density as a function of radial distance for $N=1000$.

density as a function of radial distance for this configuration. A fairly constant amplitude oscillation around the predicted constant density $3 / 4 \pi=0.2387$.. suggests that the shells are merging to form a uniformly distributed continuum. Further evidence in support of this comes from computing the two-point separation probability distribution and comparing with the Williamson probability density (2.51). The results are presented in fig. 10. The solid line is the numerically computed separation distribution and the dashed line is the Williamson probability density with $R=(N-1)^{1 / 3}$ and $N=1000$. A convergence towards a uniform distribution is clearly suggested by the data. Computing the average separation yields $\bar{r}=1.0251 R$ which is again in good agreement with the analytic result given by (2.52).

To investigate the large $N$ limit further we compute the quantities discussed above for $N=10000$. The configuration we computed in this case has energy $V=1.000022 B_{10000}$, so again it is close to the global minimum value. In fig. 11 we plot the distance from the origin of the 10000 points. In this case the individual shells have merged into a continuum distribution, except for a crust layer near the edge of the distribution where small steps can still be seen. In fig. 12 we display the density for this configuration, which is now almost constant at the expected value $3 / 4 \pi$ over a large range. In fig. 13 we compare the two-point separation distribution (solid line) with the Williamson distribution (dashed line) and find a remarkable agreement. The average separation computed from our data is $\bar{r}=1.0277 R$, again a close fit to the analytic value.

In fig. 14 we plot the distribution of nearest neighbour separations for $N=1000$ (solid 


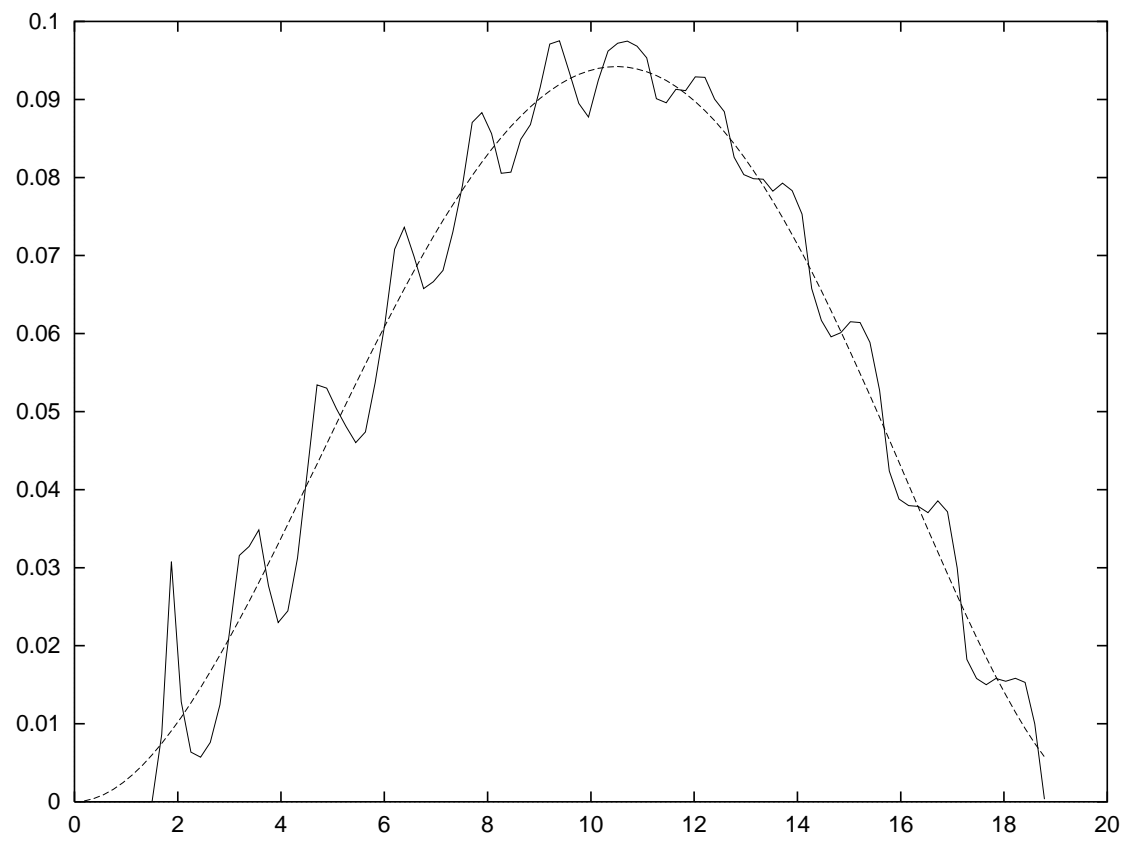

Figure 10: The two-point separation distribution for 1000 points (solid line) and the Williamson distribution (dashed line).

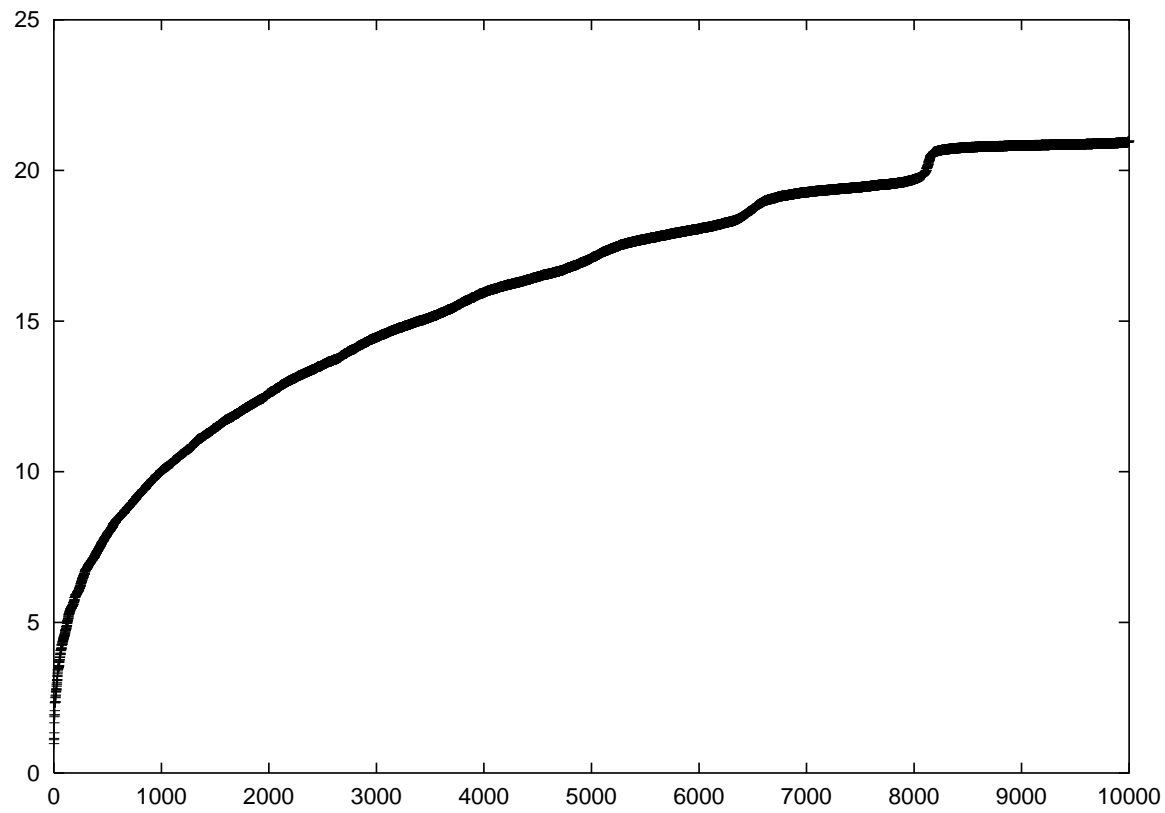

Figure 11: The distance of each point from the origin for $N=10000$. 


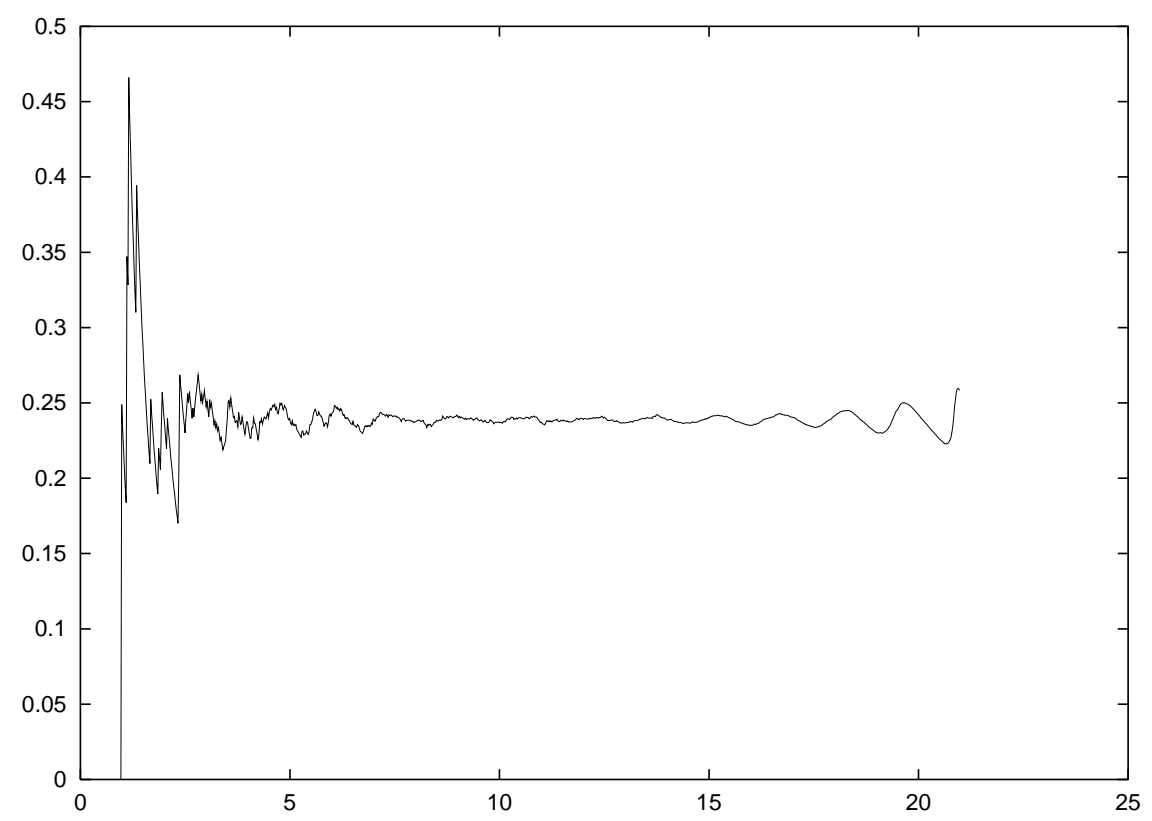

Figure 12: The density as a function of radial distance for $N=10000$.

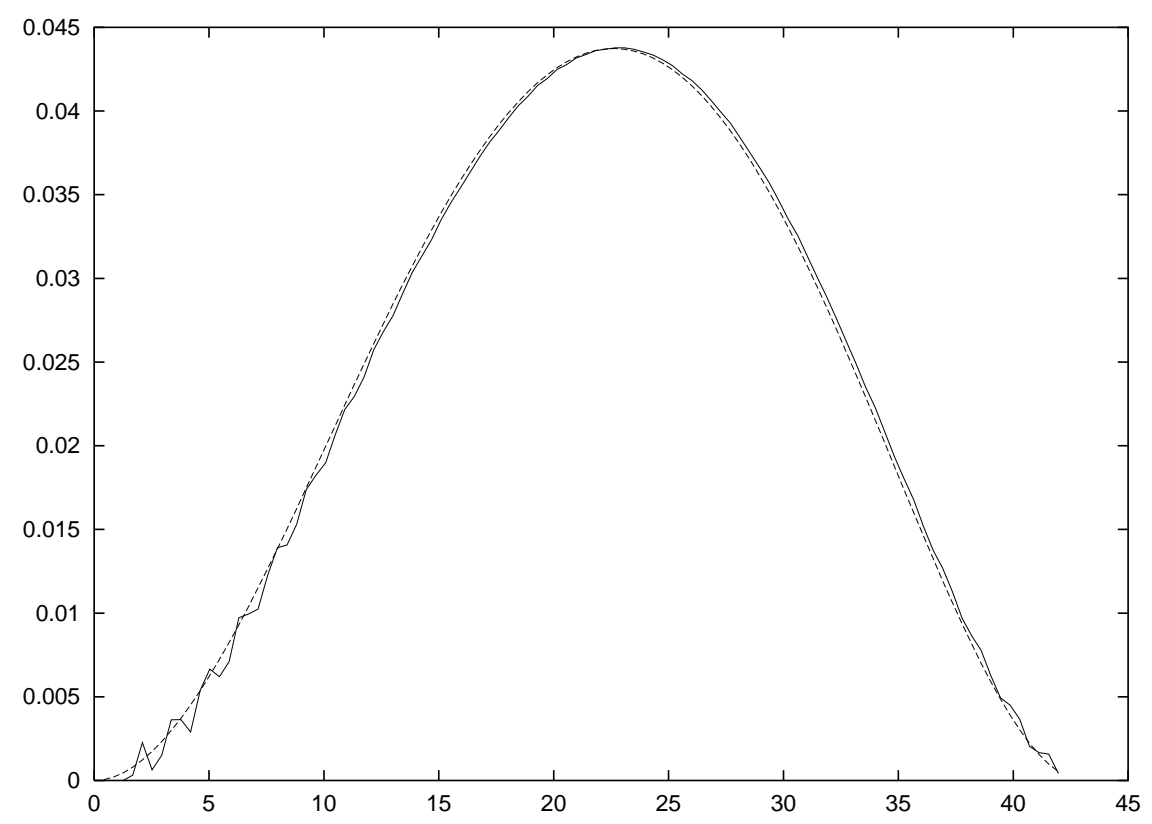

Figure 13: The two-point separation distribution for 10000 points (solid line) and the Williamson distribution (dashed line). 


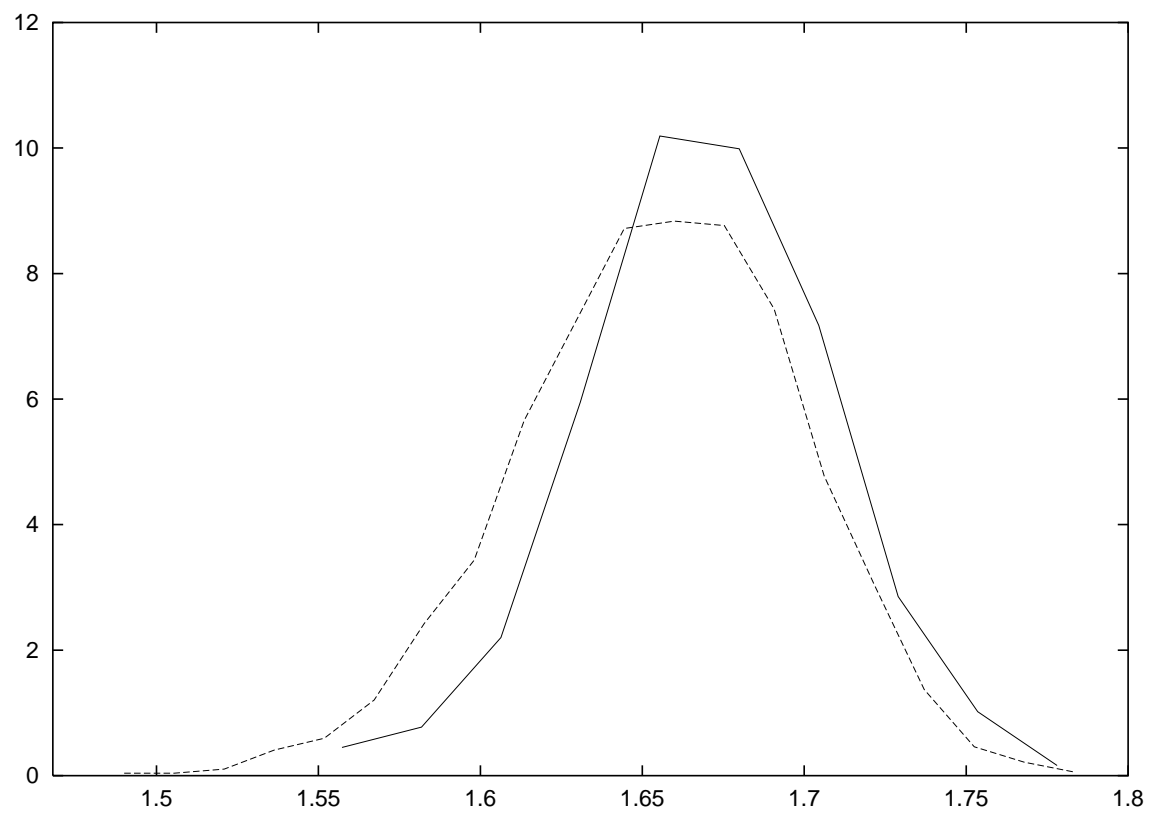

Figure 14: The distribution of nearest neighbour separations for $N=1000$ (solid line) and $N=10000$ (dashed line).

line) and $N=10000$ (dashed line). In both cases all the nearest neighbour separations $r$ satisfy $1.48<r<1.80$. The distributions are peaked around the value $r \approx 1.65$ which determined our earlier choice of the sphere packing diameter $d \approx 1.65$.

We have also computed the distribution of the angles within triangles formed from every triplet of points, analogous to a three-point function. This appears to be almost universal for all $N$ and is illustrated in fig. 15 for $N=75, N=500$ and $N=1000$. The distributions for $N=500$ and $N=1000$ are almost identical, and only when $N=75$ are there significant deviations from the universal distribution due to the effects of discreteness. We also computed the probability that the triangle was acute-angled which can be computed to be $33 / 70 \approx 0.4714$ based on the Williamson distribution. For $N=75$ we computed this probability to be 0.4981 and it was 0.4685 and 0.4743 for $N=500$ and $N=1000$ respectively, all very close to the analytic value.

All the above results are compatible with a hard sphere model, similar to Bernal's hard sphere model for liquids in which one tries to pack a sphere of radius $R$ with $N$ impenetrable spheres of diameter $d$.

\subsection{Crystallization and orientational order}

Despite the wide-spread belief that in the limit of infinite numbers of particles the minimum of the OCP model is given by a Body Centred Cubic (BCC) crystal, our preliminary results 


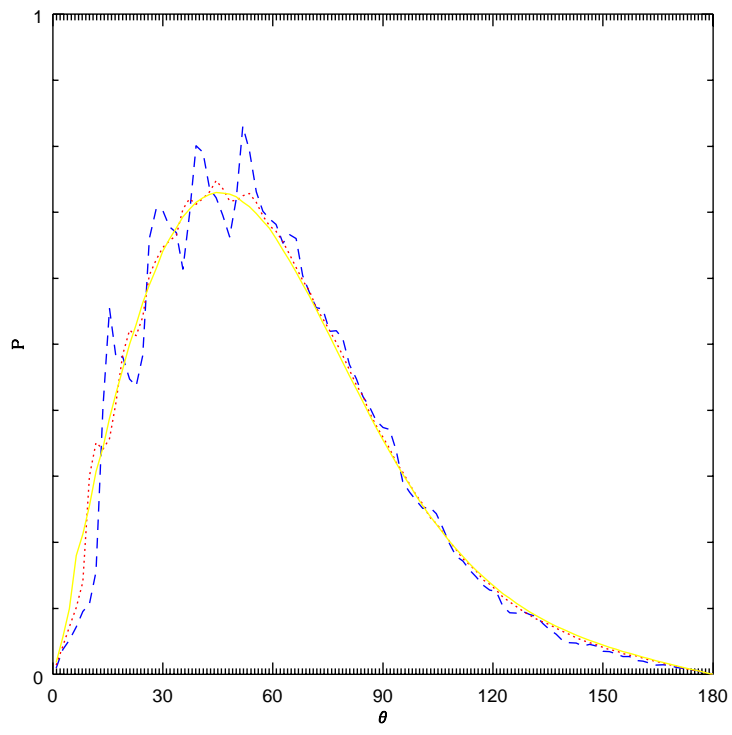

Figure 15: The probability distribution of angles within triangles in configurations with $N=1000$ (solid line), $N=500$ (dotted line) and $N=75$ (dashed line).

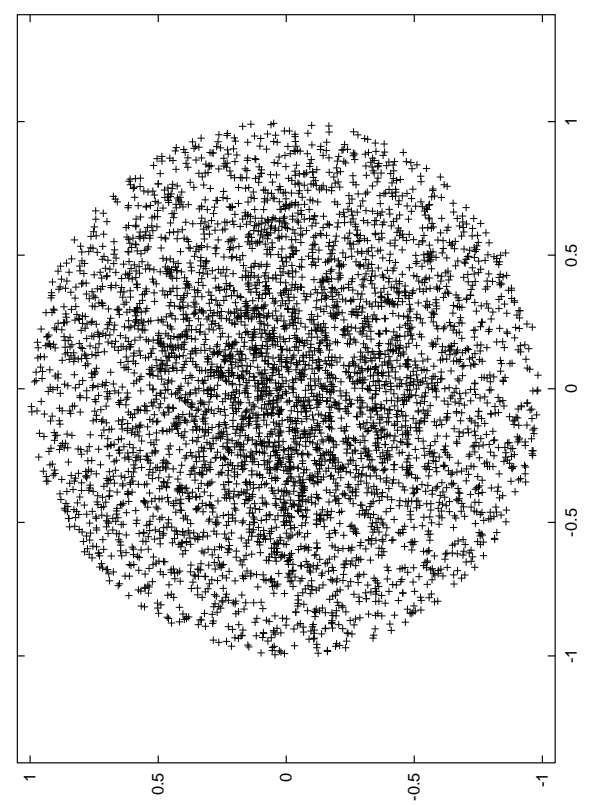

Figure 16: The distribution of nearest neighbour directions, for $N=10000$, as points on the unit sphere projected onto the unit disc. 
for up to 10,000 particles appear to show no sign of crystallization, nor orientational order. To demonstrate this we compute, for each point, the direction of its nearest neighbour. This gives a set of $N$ points on the unit sphere. In fig. 16 we display, by stereographic projection onto the unit disc in the plane, the points obtained this way which lie in the northern hemisphere (a similar plot is obtained for the southern hemisphere). After accounting for the slight distortion produced by the stereographic projection we see that these points are essentially distributed uniformly on the unit sphere. This indicates that there is no orientational order or crystal structure. We have checked that these results are not confused by any kind of a crust distribution by confirming that a similar picture is obtained by computing only with a central core of the configuration.

Of course one may always argue that our numerical method has simply not found the global minimum energy configuration, and hence we do not observe a crystal structure. It is impossible to rule out this possibility, though there are a couple of comments to be made which relate to this issue. The first is that using the same numerical codes we have studied a two-dimensional version of this problem, and found that a crystal structure does emerge and that it is numerically easy to find and display. These results will be presented elsewhere and tend to suggest that our codes should be capable of finding a crystal structure if it is truly preferred. The second point is that our numerical algorithms are based on physical processes such as thermal fluctuations, so that even if we have not found the global minima then these non-crystalline local minima should still be of physical relevance.

\subsection{The packing fraction}

The packing fraction $\eta$ of $N$ spheres of radius $a$ confined to a volume $A$ is defined by

$$
\eta=\frac{4 \pi N a^{3}}{3 A} .
$$

In our case

$$
\eta=N d^{3} / 8 R^{3} .
$$

If we assume the earlier relation that $R=(N-1)^{\frac{1}{3}}$, then for large $N$

$$
\eta \approx\left(\frac{d}{2}\right)^{3} .
$$

Substituting $d=1.65$ gives $\eta=0.56$.

It is now known that hexagonal close packing $(\mathrm{HCP})$ or face centred cubic close packing (FCC) have the densest possible value $\eta_{F C C}=0.74$. Body centred cubic (BCC) has $\eta_{B C C}=$ 0.68 , and simple cubic packing (SCC) even smaller, $\eta_{S P C}=0.52$.

Numerical and experimental data used by liquid theorists give mean values $\bar{\eta}_{R C P}=$ 0.64 [34], although the precise definition of random close packing (RCP) seems uncertain (see [28] for a recent discussion of this issue). Nevertheless, the value of $\eta$ that we have obtained is in reasonable agreement with this one. 


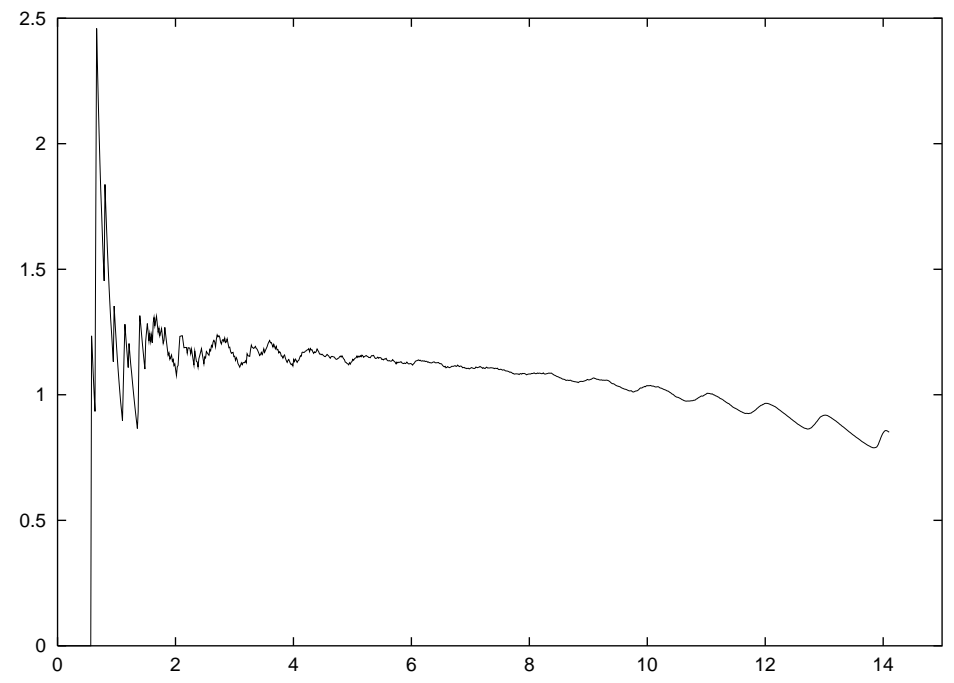

Figure 17: The density as a function of radial distance in a model where the interaction is generated by an inverse cube force with $N=10000$. Notice that the density is not constant on the outer extremities.

\section{Discussion and conclusions}

By use of numerical algorithms we have investigated in detail central configurations where the interaction force is that of an inverse square law and the masses (charges) of all the particles are equal. We find that for low values of $N$ the configurations are generally convex deltrahedra which gives way to a multi-shell structure for $N>12$. As $N$ increases the number of shells increases and eventually the configuration tends towards having a constant density. The two-point probability distribution and also the probability of acute angle triangles agree to a high degree with those of a uniform distribution. The distribution of nearest neighbours is sharply peaked suggesting that each particle can be approximated by a sphere of diameter $d \approx 1.65$ and we have found, at this stage, no evidence for longrange orientational order in contrast to the situation in 2-dimensions, which we shall present elsewhere. It still remains an open question as to whether crystallization occurs, and the possibility remains that for large values of $N$ either we may not have found a minimum sufficiently close to the global one, or that we have not probed sufficiently large values of $N$. These aspects are currently under further investigation.

The specific types of central configurations that we have computed are examples for just one of a large set of models. As we have explained, the interaction potential we have studied has a number of special properties, and we should note that different force laws will lead to very different results. To illustrate this we have included fig. 17 which shows the density distribution as a function of radial distance for particles with $N=10000$ when the interaction force is an inverse cube law. Clearly in this case there is a decreasing trend 


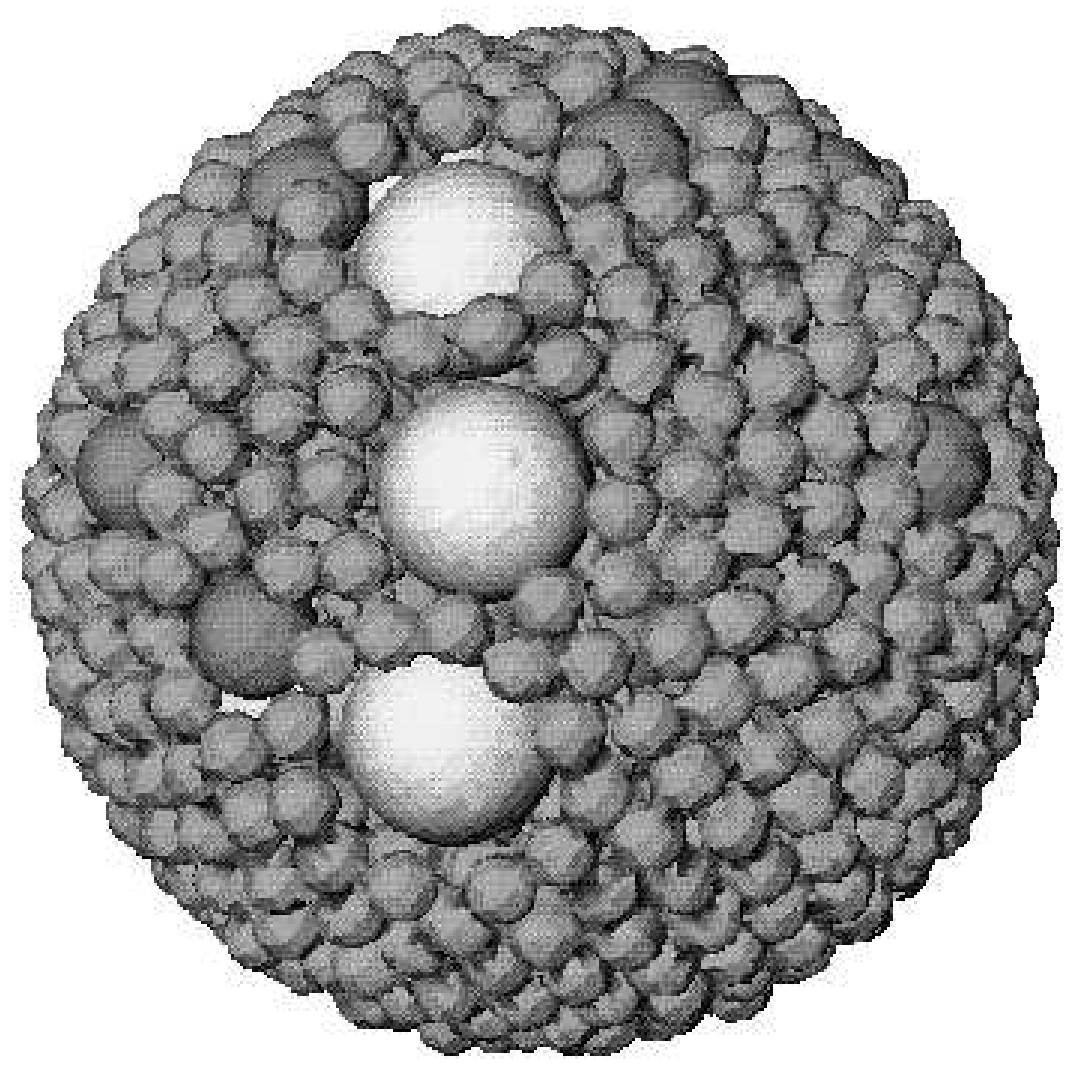

Figure 18: The distribution of 124 points, 100 of which have $m=1,20$ have $m=5$ and 4 have $m=25$. Each point with $m=1$ is represented by a sphere of diameter $d=1.65$ and the others by spheres with a diameter related to their mass by $d \propto m^{1 / 3}$. See fig18.jpg for a colour version of this figure.

in the density with increasing radius, rather than the approach to uniform density that we have encountered so far in this paper. If the power in the interaction force is further increased then this downward trend becomes even more apparent.

Another interesting possibility is to consider situations in which the particles have different masses. Using the intuition that each of the particles can be represented by a sphere, our earlier analysis suggests that the diameter of this sphere should be taken to be proportional to $m^{1 / 3}$ and indeed we find this to be the case. This is illustrated in fig. 18, where the spheres can be seen to fit snugly together using the above prescription of taking the volume of the sphere proportional to the mass of the particle.

Clearly the current work is only the tip of the iceberg in terms of the full generality of the concepts involved in central configurations, but we believe it represents a good starting point for further work. Investigations into different power laws for the interactions, differ- 
ent mass distributions and the all important question of whether crystallization occurs in these types of models are all underway.

\section{Acknowledgements}

We acknowledge advanced fellowships from PPARC (RAB) and EPSRC (PMS). We thank Mike Moore for useful discussions.

\section{Note Added}

After the submission of this paper there appeared [29] who treat larger values of $N$ than we do here. They find numerical evidence for a transition at $N=N_{c}$ to a BCC structure, where $1.1 \times 10^{4}<N_{c}<1.5 \times 10^{4}$. From this paper we became aware of earlier relevant papers including $[23,30]$ whose results for small values of $N$ have considerable overlap with our own. Where comparisons are possible, we find good agreement both qualitatively and quantitatively. Another relevant reference is [6] who list all symmetric (but not necessarily stable) solutions. Again, where comparisons are possible, their and our results agree.

\section{References}

[1] M.F. Atiyah and P.M. Sutcliffe, The geometry of point particles, Proc. R. Soc. A 458 (2002) 1089.

[2] M. Baus and J.-P. Hansen, Statistical mechanics of simple Coulomb systems, Physics Reports 59 (1980) 1.

[3] J.D. Bernal, The structure of liquids, Proc. Roy. Soc. A280 (1964) 299.

[4] A. Blimovitch, Berichte serbische Akad. Wiss. Belgrad, 59 (1927) 17.

[5] A. Blimovitch, Berichte serbische Akad. Wiss. Belgrad, 63 (1929) 1.

[6] F. Cedo and J. Llibre, Symmetric central configurations of the spatial n-body problem, J. Geom. Phys. 6 (1989) 367.

[7] T. Erber and G.M. Hockney, Complex systems: Equilibrium configurations of $N$ equal charges on a sphere, Adv. Chem. Phys. 98 (1997) 495.

[8] N. Faycal, On the classification of pyramidal central configurations, Proc. Amer. Math. Soc. 124 (1996) 249.

[9] L.L. Foldy, Electrostatic stability of Wigner and Wigner-Dyson lattices, Phys. Rev. B17 (1978) 4889. 
[10] K. Glass, Equilibrium configurations for a system of $N$ particles in the plane, Phys. Lett. A235 (1997) 591.

[11] K. Glass, Symmetry and bifurcations of planar configurations of the $N$-body and other problems, Dynamics and Stability of Systems 15 (2000) 59.

[12] D. Gregory, Isaac Newton and their Circle, ed W G Hiscock Oxford (1937) p21.

[13] Y. Hagihara, Celestial Mechanics, Volume I MIT Press.

[14] T.C. Hales, An overview of the Kepler conjecture, math.MG/9811071.

[15] G.R. Hall, Acute triangles in the n-Ball, J. Appl. Prob. 19 (1982) 712.

[16] E. Halley, Of the Infinity of the Sphere of Fix'd Stars, Phil. Trans. 31 (1720-1721) 22; Of the Order and Light of the Fix'd Stars, Phil. Trans. 31 (1720-1721) 24.

[17] W.B. Klemper, Some properties of Rosette configurations of gravitating bodies in homographic equilibrium, Astronomical Journal 67 (1962) 162.

[18] I. Kogan, A.M. Perelomov and G.W. Semenoff, Charge distribution in 2-D electrostatics, Phys. Rev. B45 (1992) 12084.

[19] R. Lehmann Filhés, Ueber zwei Fälle des Vielkörperproblems, Astr nachr 127 (1891) 137.

[20] E.H. Lieb and H. Narnhofer, The thermodynamic limit from jellium, Journal of Statistical Physics 12 (1975) 291; Erratum in 14 (1976) 465.

[21] N. Max, Another harmony of spheres, Nature 355 (1992) 115.

[22] P. Pizetti, Casi particolari del problema dei tre corpi, Rendiconti 13 (1903) 17.

[23] R. Rafac, J.P. Schiffer, J.S. Hangst, D.H.E. Dubin and D.J. Wales, Stable configurations of confined cold ionic systems, Proc. Natl. Acad. Sci. 88 (1991) 483.

[24] W.A. Rogers, On the relation between the lengths of the yard and the meter, Phys. Rev. 1 (1893) 19.

[25] D.S. Schmidt, Central configurations in $\mathbb{R}^{2}$ and $\mathbb{R}^{3}$, Contemporary Mathematics 81 (1988) 59.

[26] J.J. Thomson, On the structure of the atom, Phil Mag(6) 7 (1904) 237.

[27] J.J. Thomson, The corpuscular theory of matter, Constable (1907).

[28] S. Torquato, T.M. Truskett and P.G. Debenedetti, Is random close packing of spheres well defined?, Phys. Rev. Lett. 84 (2000) 2064. 
[29] H. Totsuji, T. Kishimoto, C. Totsuji and K. Tsuruta, Competition between two forms of ordering in finite Coulomb clusters, Phys. Rev. Lett. 88 (2002) 125002.

[30] K. Tsuruta and S. Ichimaru, Binding energy, microstructure, and shell model of Coulomb clusters, Phys. Rev. A 48 (1993) 1339.

[31] P.J.M. van Laarhoven and E.H.L. Aarts, Simulated annealing: theory and applications, Kluwer Academic Publishers, (1987).

[32] B. Williamson, An elementary treatise on the integral calculus, Longman and Green (1877).

[33] A. Winter, The analytical foundations of celestial mechanics, Princeton University Press (1941).

[34] J. Ziman, Models of Disorder, Cambridge University Press. 
This figure "fig1.jpg" is available in "jpg" format from: http://arxiv.org/ps/hep-th/0201101v2 
This figure "fig5.jpg" is available in "jpg" format from: http://arxiv.org/ps/hep-th/0201101v2 
This figure "fig18.jpg" is available in "jpg" format from: http://arxiv.org/ps/hep-th/0201101v2 